\title{
UMBILIC HYPERSURFACES OF CONSTANT SIGMA-K CURVATURE IN THE HEISENBERG GROUP
}

\author{
JIH-HSIN CHENG, HUNG-LIN CHIU, JENN-FANG HWANG, AND PAUL YANG
}

\begin{abstract}
We study immersed, connected, umbilic hypersurfaces in the Heisenberg group $H_{n}$ with $n \geq 2$. We show that such a hypersurface, if closed, must be rotationally invariant up to a Heisenberg translation. Moreover, we prove that, among others, Pansu spheres are the only such spheres with positive constant sigma-k curvature up to Heisenberg translations.
\end{abstract}

\section{Introduction and statement of the results}

In [6] we studied umbilic hypersurfaces in the Heisenberg group $H_{n}$. We proved that Pansu spheres are the only umbilic spheres with positive constant $p$ (or horizontal)mean curvature in $H_{n}$ up to Heisenberg translations. In this paper, we want to extend the result to the case of constant horizontal sigma-i curvature.

First let us define horizontal sigma-i curvature of a hypersurface $\Sigma$ in $H_{n}$. Throughout this paper, we always assume $\Sigma$ is immersed (say, $C^{2}$ smooth and $C^{\infty}$ smooth in the region of regular points, see the definition of regular point below) and $n \geq 2$. Let $\xi(J$, resp.) denote the standard contact $(C R$, resp.) structure on $H_{n}$, defined by the kernel of the contact form

$$
\Theta=d t+\sum_{j=1}^{n}\left(x_{j} d y_{j}-y_{j} d x_{j}\right)
$$

where $x_{1}, . ., x_{n}, y_{1}, . ., y_{n}, t$ are coordinates of $H_{n}$. A point $p \in \Sigma$ is called singular if $\xi=T \Sigma$ at $p$. Otherwise $p$ is called regular or nonsingular. For a regular point, we define $\xi^{\prime} \subset \xi \cap T \Sigma$ by

$$
\xi^{\prime}=(\xi \cap T \Sigma) \cap J(\xi \cap T \Sigma) .
$$

Let $\left(\xi^{\prime}\right)^{\perp}$ denote the space of vectors in $\xi$, perpendicular to $\xi^{\prime}$ with respect to the Levi metric $G:=\frac{1}{2} d \Theta(\cdot, J \cdot)=\sum_{j=1}^{n}\left[\left(d x_{j}\right)^{2}+\left(d y_{j}\right)^{2}\right]$. It is not hard to see $\operatorname{dim}(\xi \cap$ $T \Sigma) \cap\left(\xi^{\prime}\right)^{\perp}=1$. Take $e_{n} \in(\xi \cap T \Sigma) \cap\left(\xi^{\prime}\right)^{\perp}$ of unit length. Define the horizontal normal $e_{2 n}:=J e_{n}$. Let $\nabla$ denote the pseudohermitian connection associated to $(J, \Theta)$. Let $\alpha$ be the function on $\Sigma$, such that $\alpha e_{2 n}+T \in T \Sigma$ where $T:=\frac{\partial}{\partial t}$. Define $J^{\prime}$ on $\xi \cap T \Sigma$ by

$$
J^{\prime}=J \text { on } \xi^{\prime} \text { and } J^{\prime} e_{n}=0 .
$$

We now have a symmetric shape operator $\mathfrak{S}: \xi \cap T \Sigma \rightarrow \xi \cap T \Sigma$, defined by

$$
\mathfrak{S}(v)=-\nabla_{v} e_{2 n}+\alpha J^{\prime} v \text {. }
$$

1991 Mathematics Subject Classification. Primary: 35L80; Secondary: 35J70, 32V20, 53A10, 49Q10.

Key words and phrases. sigma-k curvature, Heisenberg group. 
(see Proposition 2.2 in [6]). A regular point $p$ is called an umbilic point if $\mathfrak{S}\left(\xi^{\prime}\right) \subset$ $\xi^{\prime}$ at $p$ and all the eigenvalues of $\mathfrak{S}$ acting on $\xi^{\prime}$ are the same (see also Definition 2.1 in Section 2). Let us denote this common eigenvalue by $k$. On the other hand, by Proposition 2.3 in [6], we have

$$
\mathfrak{S}\left(e_{n}\right)=l e_{n}
$$

for some real number $l$. The sigma-m curvature is the m-th symmetric function of eigenvalues of the shape operator $\mathfrak{S}$. For instance, the sigma- 1 curvature is nothing but $p$-(or horizontal) mean curvature $H$, which is the trace of $\mathfrak{S}$. In terms of $k$ and $l$, we have

$$
H=l+(2 n-2) k
$$

at an umbilic point. Similarly the sigma-i curvature, denoted as $\sigma_{i, n}$, reads

$$
\sigma_{i, n}=\left(\begin{array}{c}
2 n-2 \\
i-1
\end{array}\right) l k^{i-1}+\left(\begin{array}{c}
2 n-2 \\
i
\end{array}\right) k^{i}
$$

for $1 \leq i \leq 2 n-2$ while

$$
\sigma_{2 n-1, n}=l k^{2 n-2} .
$$

In [6], we study the case $i=1$, i.e., $\sigma_{1, n}:=H$ is a positive constant. Among others, we show that any umbilic sphere of positive constant $H$ in $H_{n}, n \geq 2$, is a Pansu sphere up to a Heisenberg translation. Let us recall what Pansu spheres are. For any $\lambda>0$, the Pansu sphere $S_{\lambda}$ is the union of the graphs of the functions $f$ and $-f$, where

$$
f(z)=\frac{1}{2 \lambda^{2}}\left(\lambda|z| \sqrt{1-\lambda^{2}|z|^{2}}+\cos ^{-1} \lambda|z|\right), \quad|z| \leq \frac{1}{\lambda} .
$$

It is known that $S_{\lambda}$ has $p$-(or horizontal) mean curvature $H=2 n \lambda$ (see Section 2 in [6] for instance).

In this paper, we will study umbilic hypersurfaces of constant general sigma-i curvature $\sigma_{i, n}$. We have the following result.

Theorem A. Let $\Sigma$ be an immersed, connected, orientable, closed, umbilic hypersurface of $H_{n}, n \geq 2$ with nonvanishing Euler number. For a given $i, 1 \leq i \leq$ $2 n-1$, suppose $\sigma_{i, n}$ of $\Sigma$ is a positive constant. Then $\Sigma$ must be a Pansu sphere up to a Heisenberg translation.

Theorem B. Let $\Sigma$ be an immersed, connected, umbilic hypersurface in $H_{n}, n$ $\geq 2$. Let $S_{\Sigma}$ denote the set of all singular points in $\Sigma$. Then

(a) either $\alpha^{2}+k^{2} \equiv 0$ on $\Sigma$ or $\alpha^{2}+k^{2}>0$ at all points in $\Sigma \backslash S_{\Sigma}$.

(b) Suppose $\alpha^{2}+k^{2} \equiv 0$ on $\Sigma$. Then $\Sigma$ is congruent with part of a hypersurface $C^{n-1} \times \gamma \times R$ where $\gamma$ is a curve in the Euclidean plane $C$ with signed curvature $l$ $(=H)$.

(c) Suppose $\alpha^{2}+k^{2}>0$ at all points in $\Sigma \backslash S_{\Sigma}$. Then $\Sigma$ is congruent with part of a rotationally invariant hypersurface. Moreover, the radius of leaves in the associated foliation is $\frac{1}{\sqrt{\alpha^{2}+k^{2}}}$.

For the situation of $\sigma_{i, n}=c \leq 0$, we have the following result.

Theorem C. Let $\Sigma$ be an immersed, connected, umbilic hypersurface of $H_{n}, n$ $\geq 2$.

(a) Suppose $\sigma_{i, n}=c=0$. Then there is no closed such hypersurface $\Sigma$. 
(b) Suppose $\sigma_{i, n}=c<0$. Then for $i$ odd, $1 \leq i \leq 2 n-1$, any closed such hypersurface $\Sigma$ must be a Pansu sphere up to a Heisenberg translation.

We remark that for the case $\sigma_{i, n}=c<0, i \geq 4$ and even, there may correspond closed umbilic hypersurfaces which are not congruent with Pansu spheres (see Figure 4.2 and Proposition 4.2). In Section 2 we give a proof of Theorem B. In Section 3 we discuss solutions to an ODE for $(\alpha, k)$ (see (3.2)). The main result is described in Theorem 3.1 and Theorem 3.3. In Section 4, among others, we apply these ODE results Theorem 3.1 and Theorem 3.3 to prove Theorem A and Theorem C. In Appendix A we derive an "almost" closed form of solutions to 3.2 .

Acknowledgments. J.-H. C. (P. Y., resp.) is grateful to Princeton University (Academia Sinica in Taiwan, resp.) for the kind hospitality. J.-H. C., H.-L. C., and J.-F. H. would like to thank the Ministry of Science and Technology of Taiwan for the support of the following research projects: MOST104-2115-M-001-011-MY2, MOST104-2115-M-008-003-MY2, and MOST104-2115-M-001-009-MY2, resp.. P. Y. would like to thank the NSF of the United States for the grant DMS 1509505. We would also like to thank Ms.Yu-Tuan Lin and Ms.Li-Yu Huang for the computer assistance to draw figures.

\section{UMBILICITY AND ROTATIONAL SYMMETRY}

Let $e_{\beta}, e_{n+\beta}:=J e_{\beta}, 1 \leq \beta \leq n$, be an orthonormal frame with respect to the Levi metric $G:=\frac{1}{2} d \Theta(\cdot, J \cdot)=\sum_{j=1}^{n}\left[\left(d x_{j}\right)^{2}+\left(d y_{j}\right)^{2}\right]$ (see Section 1). Recall that $T$ $:=\frac{\partial}{\partial t}$ is the (characteristic or Reeb) vector field satisfying $\Theta(T)=1$ and $d \Theta(T, \cdot)$ $=0$. Let $\left(p ; e_{\beta}(p), e_{n+\beta}(p), T(p)\right)$ be a moving frame, depending on $p \in H_{n}$. There exist one-forms $\omega^{a}, \omega^{2 n+1}, \omega_{a}^{b}$ such that

$$
\begin{aligned}
d p & =e_{\beta} \otimes \omega^{\beta}+e_{n+\beta} \otimes \omega^{n+\beta}+T \otimes \omega^{2 n+1} \\
d e_{\gamma} & =e_{\beta} \otimes \omega_{\gamma}{ }^{\beta}+e_{n+\beta} \otimes \omega_{\gamma}{ }^{n+\beta}+T \otimes \omega^{n+\gamma} \\
d e_{n+\gamma} & =e_{\beta} \otimes \omega_{n+\gamma}{ }^{\beta}+e_{n+\beta} \otimes \omega_{n+\gamma}{ }^{n+\beta}-T \otimes \omega^{\gamma} \\
d T & =0,
\end{aligned}
$$

where $\Theta=\omega^{2 n+1}$ and

$$
\begin{aligned}
\omega_{a}{ }^{b} & =-\omega_{b}{ }^{a}, \quad \text { for } 1 \leq a, b \leq 2 n, \\
\omega_{n+\alpha}{ }^{n+\beta} & =\omega_{\alpha}{ }^{\beta}, \omega_{\alpha}^{n+\beta}=-\omega_{n+\alpha}{ }^{\beta}, \quad \text { for } 1 \leq \alpha, \beta \leq n .
\end{aligned}
$$

The equations (2.1) are called the equations of motion. Let $\Sigma$ be a hypersurface of $H_{n}$. Recall that $H_{n}$ has a standard contact structure $\xi$ and $\mathfrak{S}$, the symmetric shape operator acting on $\xi \cap T \Sigma$ is defined by (1.1):

$$
\mathfrak{S}(v)=-\nabla_{v} e_{2 n}+\alpha J^{\prime} v
$$

for $v \in \xi \cap T \Sigma$. Recall that $\xi^{\prime}:=(\xi \cap T \Sigma) \cap J(\xi \cap T \Sigma)$. Recall that the coefficients $h_{a b}$ of the second fundamental form are defined by

$$
h_{a b}:=-G\left(\nabla_{e_{b}} e_{2 n}, e_{a}\right)
$$

for an orthonormal basis $e_{1}, . ., e_{n}, e_{n+1}:=J e_{1}, . ., e_{2 n-1}:=J e_{n-1}$ with respect to the Levi metric $G$. 
Definition 2.1. Let $\Sigma$ be a hypersurface of $H_{n}$. Suppose $p \in \Sigma$ is a regular point. We say that $p$ is an umbilic point if

(i) $\mathfrak{S}\left(\xi^{\prime}\right) \subset \xi^{\prime}$;

(ii) $\mathfrak{S}=k I$ on $\xi^{\prime}$ for some constant $k$.

where $I$ denotes the identity map.

Let us review some useful known results in $[\underline{6}$.

Proposition 2.2 (Proposition 4.1 in [6]). Suppose $\Sigma$ is an umbilic hypersurface of $H_{n}$. If $p \in \Sigma$ is a singular point, then it is isolated.

Proposition 2.3. (Proposition 4.2 in [6]). Suppose that $\Sigma$ is an umbilic hypersurface of $H_{n}$. Then, on the regular part of $\Sigma$, we have

$$
\begin{aligned}
e k & =e l=e \alpha=0, \quad \text { for all } e \in \xi^{\prime}, \\
e_{n} k & =(l-2 k) \alpha, \\
e_{n} \alpha & =k^{2}-\alpha^{2}-k l .
\end{aligned}
$$

Proposition 2.4 (Proposition 4.3 in [6]). Suppose that $\Sigma$ is an umbilic hypersurface. Then the subbundle $\xi^{\prime}$ generates a $(2 n-1)$-dimensional foliation on the regular part under the Lie bracket. In addition, the characteristic direction $e_{n}$ is always transversal to each leaf.

Definition 2.5. Let $\Sigma$ be a hypersurface in $H_{n}$. We say that $\Sigma$ is rotationally invariant if it is invariant under the group of rotations in $R^{2 n+1}$ about $t$-axis, the last coordinate axis.

Recall (see Section 2 in [6]) that the coefficients $h_{a b}$ of the second fundamental form are defined by

$$
h_{a b}:=-G\left(\nabla_{e_{b}} e_{2 n}, e_{a}\right)
$$

for an orthonormal basis $e_{1}, . ., e_{n}, e_{n+1}:=J e_{1}, . ., e_{2 n-1}:=J e_{n-1}$ with respect to the Levi metric $G$.

Theorem 2.6 (Proposition 3.1 in [6]). If $\Sigma$ is rotationally invariant, then it is umbilic.

Proof. (An argument different from the one given in the proof of Proposition 3.1 in [6]) Since $\Sigma$ is rotationally invariant, it is easy to see that $\Sigma$ has the induced foliation $\Sigma=\cup_{t} S_{\rho(t)}$, where each leaf

$$
S_{\rho(t)}=\{(z, t)|| z \mid=\rho(t)\}, \text { for some } \rho(t)>0
$$

is a sphere. Actually this foliation is just the one generated by $\xi^{\prime}=(\xi \cap T \Sigma) \cap J(\xi \cap$ $T \Sigma)$ under the Lie bracket. Taking a frame $\left\{e_{\beta}, e_{n+\beta}=J e_{\beta} \mid \beta=1, \cdots, n-1\right\}$ of $\xi^{\prime}$, we have, for $1 \leq \beta \leq n-1$,

$$
\left[e_{\beta}, e_{n+\beta}\right]=-2\left(T+\alpha e_{2 n}+\frac{h_{\beta \beta}+h_{(n+\beta)(n+\beta)}}{2} e_{n}\right), \bmod \xi^{\prime} .
$$


Since $\left[e_{\beta}, e_{n+\beta}\right]$ is tangent to a leaf, there exist a $k$ such that $\frac{h_{\beta \beta}+h_{(n+\beta)(n+\beta)}}{2}=k$, for all $\beta$ ( $k$ is independent of $\beta$ ). We proceed to compute the following Lie brackets, $\bmod \xi^{\prime}$

$$
\begin{aligned}
{\left[e_{\beta}, T+\alpha e_{2 n}+k e_{n}\right] } & =\left(e_{\beta} k-e_{n+\beta} \alpha-k h_{(n+\beta) n}-\alpha h_{n \beta}\right) e_{n} \\
{\left[e_{n+\beta}, T+\alpha e_{2 n}+k e_{n}\right] } & =\left(e_{\beta} \alpha+e_{n+\beta} k+k h_{\beta n}-\alpha h_{n(n+\beta)}\right) e_{n} .
\end{aligned}
$$

Since $\xi^{\prime}$ generates a foliation and $e_{n}$ transverses to each leaf, we have

$$
\begin{aligned}
& 0=e_{\beta} k-e_{n+\beta} \alpha-k h_{(n+\beta) n}-\alpha h_{n \beta} \\
& 0=e_{\beta} \alpha+e_{n+\beta} k+k h_{\beta n}-\alpha h_{n(n+\beta)} .
\end{aligned}
$$

Since $\Sigma$ is rotationally invariant, both $k$ and $\alpha$ are constants on each leaf, we get

$$
\begin{aligned}
& 0=k h_{(n+\beta) n}+\alpha h_{n \beta} \\
& 0=k h_{\beta n}-\alpha h_{n(n+\beta)} .
\end{aligned}
$$

This implies that $h_{n \beta}=h_{n(n+\beta)}=0$, for $1 \leq \beta \leq n-1$, which means that $e_{n}$ is an eigenvalue of the shape operator $-\nabla e_{2 n}+\alpha J^{\prime}$. (or $\left.\left(-\nabla e_{2 n}+\alpha J^{\prime}\right)\left(\xi^{\prime}\right) \subset \xi^{\prime}\right)$. Now we can assume we take a frame of $\xi^{\prime}$ such that $e_{\beta}, e_{n+\beta}$ are eigenvectors. To complete the proof, we need to show that $h_{\beta \beta}=h_{(n+\beta)(n+\beta)}=k$, for $1 \leq \beta \leq n-1$. Let $\nu=\alpha e_{2 n}+k e_{n} \in\left(\xi^{\prime \perp}\right.$, we have shown $\left[e_{\beta}, e_{n+\beta}\right]=-2(T+\nu)$, $\bmod \xi^{\prime}$, which implies that $T+\nu \in T S_{\rho(t)}$. Thus $\nu$ is uniquely determined and, in the case $S_{\rho(t)}$, we have $-\frac{J(\nu)}{|\nu|^{2}}=x_{\gamma} \stackrel{\circ}{\gamma}_{\gamma}+y_{\gamma} \stackrel{\circ}{e}_{n+\gamma}$, for all $\left(x_{\gamma}, y_{\gamma}, t\right) \in S_{\rho(t)}$. We compute $\alpha \nu+k J \nu=\left(\alpha^{2}+k^{2}\right) e_{2 n}$, hence

$$
\begin{aligned}
e_{2 n} & =\frac{\alpha \nu+k J \nu}{\alpha^{2}+k^{2}}=-\left(\alpha\left(\frac{-\nu}{|\nu|^{2}}\right)+k\left(\frac{-J \nu}{|\nu|^{2}}\right)\right) \\
& =-\left(\alpha y_{\gamma}+k x_{\gamma}\right) \stackrel{\circ}{e}_{\gamma}-\left(-\alpha x_{\gamma}+k y_{\gamma}\right) \stackrel{\circ}{e}_{n+\gamma} .
\end{aligned}
$$

Therefore, for each unit vector $e=a^{\beta} \stackrel{\circ}{e}_{\beta}+a^{n+\beta} \stackrel{\circ}{e}_{n+\beta} \in \xi^{\prime}$,

$$
-\nabla_{e} e_{2 n}=\left[\alpha a^{n+\gamma}+k a^{\gamma}\right] \stackrel{\circ}{\gamma}_{\gamma}+\left[-\alpha a^{\gamma}+k a^{n+\gamma}\right] \stackrel{\circ}{n+\gamma}_{n}
$$

hence

$$
\begin{aligned}
\left\langle-\nabla_{e} e_{2 n}, e\right\rangle & =\alpha a^{\gamma} a^{n+\gamma}+k\left(a^{\gamma}\right)^{2}-\alpha a^{\gamma} a^{n+\gamma}+k\left(a^{n+\gamma}\right)^{2} \\
& =k\left[\left(a^{\gamma}\right)^{2}+\left(a^{n+\gamma}\right)^{2}\right]=k .
\end{aligned}
$$

This completes the proof.

Now we are ready to prove Theorem B stated in Section 1.

Proof. (of Theorem B) To prove (b), note that if $\alpha \equiv 0$ and $k \equiv 0$ on an umbilic hypersurface $\Sigma$, then $H=l+(2 n-2) k=l$ is a constant on each leave of the foliation for $\Sigma$ in view of Proposition 4.2 in [6] (noting that $e l=0$ for all $e \in \xi^{\prime}$ and $\hat{e}_{2 n} l=$ $T l=0)$. Moreover, if $\alpha \equiv 0$, then $\Sigma$ is locally congruent with the hypersurface $\Sigma^{*}$ $\times I$ where $\Sigma^{*}$ is a hypersurface of $C^{n}=R^{2 n}$ (cf. Theorem 1.5 in [6]) and $I$ is an open interval of $R^{1}$.

Since $\alpha=k=0$, from the equations of motion (see 2.1 ), we have

$$
\begin{aligned}
d e_{n} & =l e_{2 n} \otimes \omega^{n}+T \otimes \omega^{2 n}, \\
d e_{2 n} & =-l e_{n} \otimes \omega^{n}-T \otimes \omega^{n} .
\end{aligned}
$$


Here we have used $\omega_{2 n}{ }^{a}(T)=\omega_{2 n}{ }^{a}\left(\hat{e}_{2 n}\right)=0$ since $\alpha=0$, and hence $\omega_{2 n}{ }^{a}=$ $-l \delta_{n}^{a} \omega^{n}$ (see Proposition 5.5 in [14]). Let $Z_{n}=\frac{1}{2}\left(e_{n}-i e_{2 n}\right), \theta^{n}=\omega^{n}+i \omega^{2 n}$, the complex version of 2.7 is

$$
d Z_{n}=i l Z_{n} \otimes \omega^{n}+\frac{1}{2} i T \otimes \theta^{\bar{n}} .
$$

Therefore, we have

$$
\begin{aligned}
d_{e_{n}} Z_{n} & =i l Z_{n}, \bmod T ; \\
d_{e} Z_{n} & =0, \bmod T, \text { for all } e \in \xi^{\prime} .
\end{aligned}
$$

On the other hand, $\alpha=k=0$ implies that, for $1 \leq \beta \leq n-1$,

$$
\left[e_{\beta}, e_{n+\beta}\right]=-2\left(T+\alpha e_{2 n}+k e_{n}\right)=-2 T, \bmod \xi^{\prime} .
$$

This means that $T$ is tangent to each leaf $L$ of the $(2 n-1)$-dimensional foliation of $\Sigma$ (see Proposition 2.4). Thus $Z_{n} \perp L$ with respect to the adapted metric. Let $\pi: H_{n} \rightarrow C^{n}$ be the projection along $T$, and $Z_{n}^{*}=\pi_{*} Z_{n}, L^{*}=\pi(L)$, we have $Z_{n}^{*} \perp L^{*}$ with respect to the standard Euclidean metric on $C^{n}$. Here $L^{*}$ constitute a $(2 n-2)$-dimensional foliation on $\Sigma^{*}$. Also, from the second equation of $(2.9)$, we see that $Z_{n}^{*}$ is a constant along each leaf $L^{*}$.

Let $\Gamma(s)$ be a characteristic curve such that $\Gamma^{\prime}(s)=e_{n}$ and let $\gamma(s)=\pi(\Gamma(s))$. We have $\gamma^{\prime}(s)=\pi_{*} e_{n}=e_{n}^{*}$. We compute the derivative of $Z_{n}^{*}$ along $\gamma(s)$. From 2.9 , we have

$$
\begin{aligned}
Z_{n}^{* \prime}(s) & =Z_{n}^{\prime}(s), \quad \bmod T \\
& =d_{e_{n}} Z_{n}, \quad \bmod T \\
& =i l Z_{n}^{*}(s),
\end{aligned}
$$

which implies that

$$
Z_{n}^{*}(s)=e^{i\left[l(s)-l\left(s_{0}\right)\right]} Z_{n}^{*}\left(s_{0}\right), \text { for a fixed } s_{0} .
$$

Therefore $\gamma$ completely lies on the complex line spanned by the complex vector $Z_{n}^{*}\left(s_{0}\right)$. After an unitary transformation, we can assume, without loss of generality, that this complex line is spanned by the last coordinates $z_{n}$. Since $Z_{n}^{*}\left(s_{0}\right) \perp L^{*}\left(s_{0}\right)$, we see that $L^{*}\left(s_{0}\right)$ is an open subset of $C^{n-1}$ with coordinates $\left(z_{1}, \cdots, z_{n-1}\right)$. Thus we get $\Sigma^{*}=L^{*}\left(s_{0}\right) \times \gamma$ with $L^{*}\left(s_{0}\right) \subset C^{n-1}$ an open subset, $\gamma \subset C^{1}$, hence $\Sigma=L^{*}\left(s_{0}\right) \times \gamma \times I$.

Finally, we compute

$$
\begin{aligned}
l & =\left\langle\nabla_{e_{n}} e_{n}, e_{2 n}\right\rangle=\left\langle d_{e_{n}} e_{n}, e_{2 n}\right\rangle \\
& =\left\langle\frac{d}{d s} \Gamma^{\prime}(s), e_{2 n}\right\rangle=\frac{d}{d s} \gamma^{\prime}(s) \cdot \pi_{*} e_{2 n},
\end{aligned}
$$

where $\cdot$ is the standard inner product on the Euclidaen plane $C^{1}$. Since $\pi_{*} e_{2 n}=\hat{J} \gamma^{\prime}$, where $\hat{J}$ is the complex structure on $C^{1}$, we see that it is normal to $\gamma$, thus, from (2.12),$l$ is just the signed curvature of $\gamma$ with respect to the orthonormal frame field $\left\{\pi_{*} e_{n}, \pi_{*} e_{2 n}\right\}$. We have proved (b).

Next we are going to prove (c). On $\Sigma \backslash S_{\Sigma}$, we define

$$
N=\frac{\alpha e_{n}-k e_{2 n}}{\sqrt{\alpha^{2}+k^{2}}}
$$


(note that $\alpha^{2}+k^{2}>0$ on $\Sigma \backslash S_{\Sigma}$ by assumption). We will show that $N$ is the unit horizontal normal of each leaf $L$ in the following sense

$$
\left.d\left(X-\frac{N}{\sqrt{\alpha^{2}+k^{2}}}(X)\right)\right|_{L}=0
$$

where $X$ denotes the position vector. Since $L$ is spanned by $e_{\gamma}, e_{n+\gamma}$ and their Lie brackets, we see that 2.14 is equivalent to

$$
\begin{aligned}
& 0=d_{e_{\beta}}\left(X+\frac{-\alpha e_{n}+k e_{2 n}}{\alpha^{2}+k^{2}}(X)\right) \\
& 0=d_{e_{n+\beta}}\left(X+\frac{-\alpha e_{n}+k e_{2 n}}{\alpha^{2}+k^{2}}(X)\right) .
\end{aligned}
$$

Here $d_{v} X$ means differentiation of $X$ in the direction $v$. Note that $d_{v} X=v$. Now we are going to show 2.15): By the motion equation, for each $\gamma, 1 \leq \gamma \leq n-1$, we have

$$
\begin{aligned}
d_{e_{\gamma}} e_{n} & =\left\langle e_{\beta} \otimes \omega_{2 n}{ }^{n+\beta}-e_{n+\beta} \otimes \omega_{2 n}{ }^{\beta}+T \otimes \omega^{2 n}, e_{\gamma}\right\rangle \\
& =-\sum_{\beta=1}^{n-1} h_{(n+\beta) \gamma} e_{\beta}+\sum_{\beta=1}^{n} h_{\beta \gamma} e_{n+\beta} \\
& =-h_{(n+\gamma) \gamma} e_{\gamma}+h_{\gamma \gamma} e_{n+\gamma} \\
& =\alpha e_{\gamma}+k e_{n+\gamma} .
\end{aligned}
$$

Similarly we have

$$
\begin{aligned}
d_{e_{n+\gamma}} e_{n} & =-k e_{\gamma}+\alpha e_{n+\gamma} \\
d_{e_{\gamma}} e_{2 n} & =-k e_{\gamma}+\alpha e_{n+\gamma} \\
d_{e_{n+\gamma}} e_{2 n} & =\alpha e_{\gamma}-k e_{n+\gamma} .
\end{aligned}
$$

Recall that both $\alpha$ and $k$ are constants on each leaf, hence, using formula (2.16), (2.17), we have (2.15), hence (2.14). The formula 2.14) means that for each leaf $L$, there exists a constant $X_{L} \in H_{n}$ such that

$$
X-X_{L}=\frac{N}{\sqrt{\alpha^{2}+k^{2}}}(X) \text {, for all } X \in L .
$$

We write $\frac{N}{\sqrt{\alpha^{2}+k^{2}}}(X)=\sum_{j=1}^{2 n} a_{j}(X) \stackrel{\circ}{e}_{j}(X)$. Here $\stackrel{\circ}{j}_{j}(X):=\frac{\partial}{\partial x_{j}}+x_{j}^{\prime} T$ in which $x_{n+j}$ $=y_{j}, x_{j}^{\prime}=y_{j}$, and $x_{n+j}^{\prime}=-x_{j}$ for $1 \leq j \leq n$. Then we have

$$
\begin{aligned}
& \sum_{j=1}^{2 n} a_{j}(X)\left(x_{j}^{\prime}-\left(X_{L}\right)_{j}^{\prime}\right) \\
= & G\left(X-X_{L}, J\left(X-X_{L}\right)\right)=0
\end{aligned}
$$


in view of (2.18), and hence

$$
\begin{aligned}
& \sum_{j=1}^{2 n} a_{j}(X) \stackrel{\circ}{e}_{j}(X) \\
= & \sum_{j=1}^{2 n} a_{j}(X)\left(\frac{\partial}{\partial x_{j}}+x_{j}^{\prime} T\right) \\
= & \sum_{j=1}^{2 n} a_{j}(X)\left(\frac{\partial}{\partial x_{j}}+\left(X_{L}\right)_{j}^{\prime} T\right)+\sum_{j=1}^{2 n} a_{j}(X)\left(x_{j}^{\prime}-\left(X_{L}\right)_{j}^{\prime}\right) T \\
= & \left.\sum_{j=1}^{2 n} a_{j}(X)\left(\frac{\partial}{\partial x_{j}}+\left(X_{L}\right)_{j}^{\prime} T\right)+0(\text { by } 2.19)\right) \\
= & \sum_{j=1}^{2 n} a_{j}(X) \stackrel{\circ}{e}_{j}\left(X_{L}\right),
\end{aligned}
$$

meaning $L$ is the sphere with radius $\frac{1}{\sqrt{\alpha^{2}+k^{2}}}$ and center $X_{L}$. Finally, we will show that

$$
-\frac{k}{\alpha^{2}+k^{2}} T=d_{e_{n}}\left(X+\frac{-\alpha e_{n}+k e_{2 n}}{\alpha^{2}+k^{2}}(X)\right)=d_{e_{n}}\left(X_{L}\right) .
$$

This equation says that the center $X_{L}$ lies on a line parallel to the $t$-axis. Therefore, $\Sigma \backslash S_{\Sigma}$ (and hence $\Sigma$, since $\Sigma \backslash S_{\Sigma}$ is open and dense in $\Sigma$ by Theorem D in [12]) is congruent with a rotationally invariant hypersurface. For (2.21), we compute, as we just did for 2.16,

$$
\begin{aligned}
d_{e_{n}} e_{n} & =\left\langle e_{\beta} \otimes \omega_{n}{ }^{\beta}-e_{n+\beta} \otimes \omega_{2 n}{ }^{\beta}+T \otimes \omega^{2 n}, e_{n}\right\rangle \\
& =-\sum_{\beta=1}^{n-1} h_{(n+\beta) n} e_{\beta}+\sum_{\beta=1}^{n} h_{\beta n} e_{n+\beta} \\
& =l e_{2 n},
\end{aligned}
$$

Similarly

$$
d_{e_{n}} e_{2 n}=-l e_{n}-T \text {. }
$$

And, using 2.4, we have

$$
\begin{aligned}
& e_{n}\left(\frac{\alpha}{\alpha^{2}+k^{2}}\right)=d_{e_{n}}\left(\frac{\alpha}{\alpha^{2}+k^{2}}\right)=\frac{k^{2}+\alpha^{2}-k l}{\alpha^{2}+k^{2}}, \\
& e_{n}\left(\frac{k}{\alpha^{2}+k^{2}}\right)=d_{e_{n}}\left(\frac{k}{\alpha^{2}+k^{2}}\right)=\frac{l \alpha}{\alpha^{2}+k^{2}} .
\end{aligned}
$$

Therefore, by the motion equation and formula $2.22,2.23$ and $(2.24)$, we obtain (2.21). This completes the proof of (c).

To prove (a), suppose the converse holds. Then there exists a sequence of $p_{j} \in \in . \Sigma \backslash S_{\Sigma}$ with $\alpha_{j}^{2}+k_{j}^{2}>0$, converging to $p_{\infty} \in \Sigma$ with $\alpha_{\infty}^{2}+k_{\infty}^{2}=0$. Here we denote $\alpha\left(p_{j}\right)$ and $k\left(p_{j}\right)$ by $\alpha_{j}$ and $k_{j}$, resp. for $j=1,2, . ., \infty$. Consider the vector

$$
v_{j}:=p_{j}-X_{L_{j}}
$$


where $L_{j}$ denotes the leaf through $p_{j}$ with the center $X_{L_{j}}$. From 2.18 and 2.20), we learn that $v_{j}$ is sitting in the contact plane passing through $X_{L_{j}}$ for all $j$ and

$$
\left\|v_{j}\right\|_{G}:=\sqrt{G\left(v_{j}, v_{j}\right)}=\frac{1}{\sqrt{\alpha_{j}^{2}+k_{j}^{2}}}
$$

(recall that $G$ denotes the Levi metric). As $j \rightarrow \infty,\left\|v_{j}\right\|_{G}$ goes to infinity since $\sqrt{\alpha_{j}^{2}+k_{j}^{2}}$ tends to zero in 2.25 . So by $2.21, X_{L_{j}}$ must go to infinity along a line parallel to the $t$-axis as $j \rightarrow \infty$.On the other hand, we compute

$$
\begin{aligned}
0 & =\Theta\left(v_{j}\right)=d t\left(v_{j}\right)+\sum_{k=1}^{n}\left(x_{k}\left(X_{L_{j}}\right) d y_{k}-y_{k}\left(X_{L_{j}}\right) d x_{k}\right)\left(v_{j}\right) \\
& \rightarrow \pm \infty+\text { a bounded number }
\end{aligned}
$$

as $j \rightarrow \infty$ since $p_{j}$ goes to a (finite) point $p_{\infty}$ and the projection of $X_{L_{j}}$ on the $x y$-hyperplane is a fixed vector. The contradiction obtained from 2.26 concludes (a).

\section{An ODE SYSTEM}

From (2.4) (Proposition 4.2 in [6]), we have the following equations for $(\alpha, k)$ :

$$
\begin{aligned}
e_{n} k & =(l-2 k) \alpha, \\
e_{n} \alpha & =k^{2}-\alpha^{2}-k l
\end{aligned}
$$

on an umbilic hypersurface $\Sigma$ of $H_{n}$. Let $s$ be the unit speed parameter for integral curves of $e_{n}$. Write $k^{\prime}=\frac{d k}{d s}=e_{n} k$ and $\alpha^{\prime}=\frac{d \alpha}{d s}=e_{n} \alpha$. Then we can write 3.1. as follows:

$$
\begin{aligned}
k^{\prime} & =(l-2 k) \alpha, \\
\alpha^{\prime} & =k^{2}-\alpha^{2}-k l .
\end{aligned}
$$

Suppose $\sigma_{i, n}=c$, a constant. By $(1.2)$, this means that $k$ and $l$ satisfy the following relation:

$$
\left(\begin{array}{c}
2 n-2 \\
i-1
\end{array}\right) l k^{i-1}+\left(\begin{array}{c}
2 n-2 \\
i
\end{array}\right) k^{i}=c .
$$

Consider the points where $l-2 k=0$. We then get

$$
k^{i}=\frac{i}{2 n+i-1} \frac{c}{\left(\begin{array}{c}
2 n-2 \\
i-1
\end{array}\right)}
$$

for $1 \leq i \leq 2 n-1$. For $c>0$ we have one real root $k_{c, 2}$ to (3.4) if $i$ is odd while two real roots $\pm k_{c, 2}$ to 3.4 if $i$ is even, where

$$
k_{c, 2}=\left[\frac{i}{2 n+i-1} \frac{c}{\left(\begin{array}{c}
2 n-2 \\
i-1
\end{array}\right)}\right]^{1 / i} .
$$

We claim that the straight line $k=k_{c, 2}$ (two straight lines $k= \pm k_{c, 2}$, resp.) in the $(\alpha, k)$-plane is a solution (are solutions, resp.) to (3.2) for $i$ odd (even, resp.). When $k=k_{c, 2}\left(k= \pm k_{c, 2}\right.$, resp.), we have $l-2 k=0$ and hence $k=k_{c, 2}(k=$ 
$\pm k_{c, 2}$, resp.) satisfies the equation $k^{\prime}=(l-2 k) \alpha=0$. We can solve the second equation of 3.2 for $\alpha$ :

$$
\begin{aligned}
\alpha(s) & =k_{c, 2} \tan \left\{\tan ^{-1}\left(\frac{\alpha\left(s_{0}\right)}{k_{c, 2}}\right)+k_{c, 2}\left(s_{0}-s\right)\right\} \\
(\alpha(s) & \left.= \pm k_{c, 2} \tan \left\{\tan ^{-1}\left(\frac{\alpha\left(s_{0}\right)}{ \pm k_{c, 2}}\right) \pm k_{c, 2}\left(s_{0}-s\right)\right\}, \text { resp. }\right)
\end{aligned}
$$

where $s_{0}<s$. For $i$ odd (even, resp.), in $R^{2} \backslash\left\{k=k_{c}\right\}\left(R^{2} \backslash\left\{k= \pm k_{c, 2}\right\}\right.$, resp.), there are two (three, resp.) stationary points on the $k$-axis $(\alpha=0, k(k-l)=0)$ for the ODE system (3.1):

$$
\begin{aligned}
& (0,0) \text { and }\left(0, k_{c, 1}\right) \\
& \left((0,0) \text { and }\left(0, \pm k_{c, 1}\right), \text { resp. }\right)
\end{aligned}
$$

where $k_{c, 1}$ is a positive number such that $l-k=0$. By $(3.3)$ we get

$$
k^{i}=\frac{i}{2 n-1} \frac{c}{\left(\begin{array}{c}
2 n-2 \\
i-1
\end{array}\right)},
$$

and hence

$$
k_{c, 1}=\left[\frac{i}{2 n-1} \frac{c}{\left(\begin{array}{c}
2 n-2 \\
i-1
\end{array}\right)}\right]^{1 / i} .
$$

We are going to prove a similar result as in Lemma 6.1 of $[6]$.

Theorem 3.1. Assume $\sigma_{i, n}=c$, a positive constant. For $i$ odd and $i \geq 3$ ( $i$ even and $i \geq 2$, resp.), given any initial point $p_{0}=\left(\alpha_{0}, k_{0}\right)$

(a) Suppose $p_{0} \in\left\{k>k_{c, 2}\right\} \backslash\left\{\left(0, k_{c, 1}\right)\right\} \quad\left(\left\{|k|>k_{c, 2}\right\} \backslash\left\{\left(0, \pm k_{c, 1}\right)\right\}\right.$, resp. $)$, there passes a unique periodic orbit $\gamma \subset\left\{k>k_{c, 2}\right\} \backslash\left\{\left(0, k_{c, 1}\right)\right\} \quad\left(\left\{|k|>k_{c, 2}\right\} \backslash\left\{\left(0, \pm k_{c, 1}\right)\right\}\right.$, resp.), described by $(\alpha(s), k(s)), 0 \leq s \leq s_{0}$, which is a solution to the ODE system (3.2), with $\alpha\left(s_{0}\right)=\alpha(0)=\alpha_{0}$ and $k\left(s_{0}\right)=k(0)=k_{0}$.

(b) Suppose $p_{0} \in\left\{0<k<k_{c, 2}\right\}\left(\left\{|k|<k_{c, 2}, k \neq 0\right\} \backslash\{(0,0)\}\right.$, resp. $)$, there passes a unique arc-type orbit $\gamma \subset\left\{0<k<k_{c, 2}\right\}\left(\left\{|k|<k_{c, 2}\right\} \backslash\{(0,0)\}\right.$, resp.), described by $(\alpha(s), k(s))$, for $s \in\left(-s_{1},+s_{2}\right)$ with $s_{1}>0, s_{2}>0$, which is a solution to the ODE system (3.2), with $(\alpha(0), k(0))=p_{0}$ and $\lim _{s \rightarrow+s_{2}}(\alpha(s), k(s))=\left(-\alpha_{1}, 0\right)$ and $\lim _{s \rightarrow-s_{1}}(\alpha(s), k(s))=\left(+\alpha_{1}, 0\right)$ for some $\alpha_{1}>0$. Moreover, we have

$$
\lim _{s \rightarrow-s_{1}} \frac{d k}{d s}=+\infty, \lim _{s \rightarrow+s_{2}} \frac{d k}{d s}=-\infty .
$$

(c) Suppose $p_{0} \in\{k<0\}$ there passes a unique arc-type orbit $\gamma \subset\{k<0\}$, described by $(\alpha(s), k(s))$, for all $s \in R$, which is a solution to the ODE system 3.2), with $(\alpha(0), k(0))=p_{0}$ and $\lim _{s \rightarrow \pm \infty}(\alpha(s), k(s))=\left( \pm \alpha_{2}, 0\right)$ for some $\alpha_{2}>$ 0 .

Moreover, $\gamma$ is symmetric with respect to the $k$-axis, i.e., $(\alpha, k) \in \gamma$ implies $(-\alpha, k) \in \gamma$.

There are important curves $\Psi_{0}^{ \pm}$in $(\alpha, k)$-plane, defined by $k^{2}-\alpha^{2}-k l=0$ (where $\alpha^{\prime}=0$ ), in $k \gtrless 0$ regions, resp. (see Figure 3.1). Recall that $l$ is a function 
of $k$ by 3.3 :

$$
l=\frac{c}{\left(\begin{array}{c}
2 n-2 \\
i-1
\end{array}\right) k^{i-1}}-\frac{2 n-i-1}{i} k
$$

for $1 \leq i \leq 2 n-1$ (we have used the convention $\left(\begin{array}{c}m \\ 0\end{array}\right)=1$ ). Compute

$$
\frac{d l}{d k}=-(i-1) \frac{c}{\left(\begin{array}{c}
2 n-2 \\
i-1
\end{array}\right) k^{i}}-\frac{2 n-i-1}{i} .
$$

Expressing $\alpha$ in $k$ from $k^{2}-\alpha^{2}-k l=0$, we get

$$
\alpha=k \sqrt{\frac{2 n-1}{i}-\frac{c}{\left(\begin{array}{c}
2 n-2 \\
i-1
\end{array}\right) k^{i}}}
$$

in the region: $\alpha>0$ and $k>k_{c, 1}$. Now write $a=\frac{2 n-1}{i}, b=\frac{c}{\left(\begin{array}{c}2 n-2 \\ i-1\end{array}\right)}$. A direct computation shows

$$
\begin{aligned}
\frac{d^{2} \alpha}{d k^{2}} & =\frac{b i k^{-i-1}}{2 \sqrt{a-b k^{-i}}}+\frac{-b i^{2} k^{-i-1}}{2 \sqrt{a-b k^{-i}}}-\frac{b^{2} i^{2} k^{-2 i-1}}{4\left(a-b k^{-i}\right)^{3 / 2}} \\
& <0 \quad\left(\text { since } i^{2} \geq i\right) .
\end{aligned}
$$

From the above argument and symmetry in $k$-axis, we have shown the following result.

Lemma 3.1. $\Psi_{0}^{+}$is convex upward in the region: $\alpha>0(\alpha<0$, resp. $)$ and $k$ $>k_{c, 1}$, viewed as a graph over $k$-axis. Moreover, $\alpha$ goes like $\sqrt{\frac{2 n-1}{i}} k\left(-\sqrt{\frac{2 n-1}{i}} k\right.$ in the $\alpha<0$ region, resp.) as $k$ tends to $+\infty$.

We are going to compute curvature of a solution curve $(\alpha(s), k(s))$ to $(3.2)$ :

$$
\frac{d}{d s}\left(\tan ^{-1} \frac{k^{\prime}}{\alpha^{\prime}}\right)=\frac{k^{\prime \prime} \alpha^{\prime}-\alpha^{\prime \prime} k^{\prime}}{\left(\alpha^{\prime}\right)^{2}+\left(k^{\prime}\right)^{2}} .
$$

Taking derivatives of 3.2 and substituting into $k^{\prime \prime} \alpha^{\prime}-\alpha^{\prime \prime} k^{\prime}$, we get

$$
\begin{aligned}
& k^{\prime \prime} \alpha^{\prime}-\alpha^{\prime \prime} k^{\prime} \\
= & -(2 k-l)\left\{k ^ { 2 } \alpha ^ { 2 } \left[\left(\frac{2 n-1}{i^{2}}\right)(2 n+3 i-1)\right.\right. \\
& \left.+k^{-i} \tilde{\sigma}_{i, n}\left(\frac{-4 n+i^{2}-3 i+2}{i}\right)+k^{-2 i}\left(\tilde{\sigma}_{i, n}\right)^{2}\right] \\
& \left.+\alpha^{4}\left[\frac{2 n-i-1}{i}+(i-1) \tilde{\sigma}_{i, n} k^{-i}\right]+\left(\alpha^{\prime}\right)^{2}\right\} .
\end{aligned}
$$

In deriving (3.7) we have used 3.6 and let

$$
\tilde{\sigma}_{i, n}=\frac{\sigma_{i, n}}{\left(\begin{array}{c}
2 n-2 \\
i-1
\end{array}\right)}
$$

(see the expression of $\sigma_{i, n}$ in $(1.2)$ ). Denote the main term in (3.7) by

$$
\begin{aligned}
\Pi: & =k^{2} \alpha^{2}\left[\left(\frac{2 n-1}{i^{2}}\right)(2 n+3 i-1)\right. \\
& \left.+k^{-i} \tilde{\sigma}_{i, n}\left(\frac{-4 n+i^{2}-3 i+2}{i}\right)+k^{-2 i}\left(\tilde{\sigma}_{i, n}\right)^{2}\right] \\
& +\alpha^{4}\left[\frac{2 n-i-1}{i}+(i-1) \tilde{\sigma}_{i, n} k^{-i}\right]+\left(\alpha^{\prime}\right)^{2}
\end{aligned}
$$


where $\alpha^{\prime}$ in the last term should mean $k^{2}-\alpha^{2}-k l$ by (3.2). So from (3.7) and 3.8 , we can write

$$
\begin{aligned}
\frac{d^{2} \alpha}{d k^{2}} & =\frac{d}{d k}\left(\frac{\alpha^{\prime}}{k^{\prime}}\right) \\
& =\frac{\alpha^{\prime \prime} k^{\prime}-\alpha^{\prime} k^{\prime \prime}}{\left(k^{\prime}\right)^{3}}=\frac{(2 k-l) \Pi}{\left(k^{\prime}\right)^{3}} \\
& =-\frac{\Pi}{(2 k-l)^{2} \alpha^{3}}
\end{aligned}
$$

by 3.2 .

Proof. (of Theorem 3.1) If the initial point $p_{0}$ is in $k$-axis with $k_{0}>k_{c, 1}$, then $k^{\prime}(0)=0$ and $\alpha^{\prime}(0)>0$. So according to the ODE (3.2), $p_{0}$ is moving into the region surrounded by the line segment $\{0\} x\left[k_{c, 1}, \infty\right)$ and the curve $\Psi_{0}^{+} \cap\{\alpha>0\}$ in small positive time. Call this region $I^{+}$(see Figure 3.1). Since the solution to (3.2) is symmetric with respect to the $k$-axis, we need only to discuss the $\alpha$-positive part. We may assume $i \geq 2$ in the following argument (for the case $i=1, \sigma_{1, n}$ is the $p$-mean curvature. We refer the reader to [6]).

Case 1. Suppose $p_{0} \in$ Region $I^{+}$. From the equation 3.2 we learn that $k^{\prime}=$ $(l-2 k) \alpha<0$ since $\alpha>0$ and $l-2 k<0$ for $k>k_{c, 1}$. On the other hand, $\alpha^{\prime}=$ $k^{2}-\alpha^{2}-k l>0$ since $p_{0}$ is above the curve $\Psi_{0}^{+}$. Altogether we have

$$
\frac{d k}{d \alpha}=\frac{k^{\prime}}{\alpha^{\prime}}=\frac{(l-2 k) \alpha}{k^{2}-\alpha^{2}-k l}<0
$$

in Region $I^{+}$. It follows from 3.10$)$ and Lemma 3.1 that the solution $(\alpha(s), k(s))$ to (3.2) with $(\alpha(0), k(0))=p_{0}$ must hit $\Psi_{0}^{+}$in finite positive time. That is, for some $s_{1}>0,\left(\alpha\left(s_{1}\right), k\left(s_{1}\right)\right) \in \Psi_{0}^{+}$. Next what happens as $s \rightarrow-\infty$ ? In view of 3.10 we have $(\alpha(s), k(s)) \in \Omega_{0}:=\left[0, \alpha_{0}\right] \times\left[k_{0}, \infty\right)$ for $s \leq 0$. There exists large $k_{1}>>k_{c, 1}$ such that for $k>k_{1},|\alpha| \leq \alpha_{0}$, we have $2 k-l=O(k), k^{2}-k l \geq k^{2}$, and hence

$$
\begin{aligned}
\left|\frac{d k}{d \alpha}\right| & =\left|\frac{k^{\prime}}{\alpha^{\prime}}\right| \\
& =\left|\frac{(l-2 k) \alpha}{k^{2}-\alpha^{2}-k l}\right| \\
& \leq \frac{C_{1}}{k} \leq C_{2}
\end{aligned}
$$

in $\Omega_{0}$. Therefore $(\alpha(s), k(s))$ must hit $\{0\} \times\left(k_{c, 1}, \infty\right)$ in negative finite time. That is, for some $s_{2}<0,\left(\alpha\left(s_{2}\right), k\left(s_{2}\right)\right) \in\{0\} \times\left(k_{c, 1}, \infty\right)$.

If $p_{0} \in \Psi_{0}^{+} \cap\{\alpha>0\}, \alpha^{\prime}(0)=0$ and $k^{\prime}(0)=(l-2 k)(0) \alpha(0)<0$ since $(l-2 k)(0)$ $<0, \alpha(0)>0$. So by Lemma 3.1, $p_{0}$ will move into the region $I I^{+}$surrounded by the curve $\Psi_{0}^{+} \cap\{\alpha>0\}$, line segment $\{0\} \times\left[k_{c, 2}, k_{c, 1}\right]$, and the horizontal line segment $L_{c, 2}^{+}:=\left\{\left(\alpha, k_{c, 2}\right): \alpha \geq 0\right\}$ in small positive time.

Case 2. Suppose $p_{0} \in$ Region $I I^{+}$. Write Region $I I^{+}=$Region $I I_{1} \cup$ the horizontal line segment $L_{c, 1}^{+}:=\left\{\left(\alpha, k_{c, 1}\right): \alpha \geq 0\right\} \cup$ Region $I I_{2}$. Here Region $I I_{1}$ denotes the region surrounded by the curve $\Psi_{0}^{+} \cap\{\alpha>0\}$ and $L_{c, 1}^{+}$while Region $I I_{2}$ denotes the region surrounded by $L_{c, 1}^{+}$, line segment $\{0\} \times\left[k_{c, 2}, k_{c, 1}\right]$, and $L_{c, 2}^{+}$. 


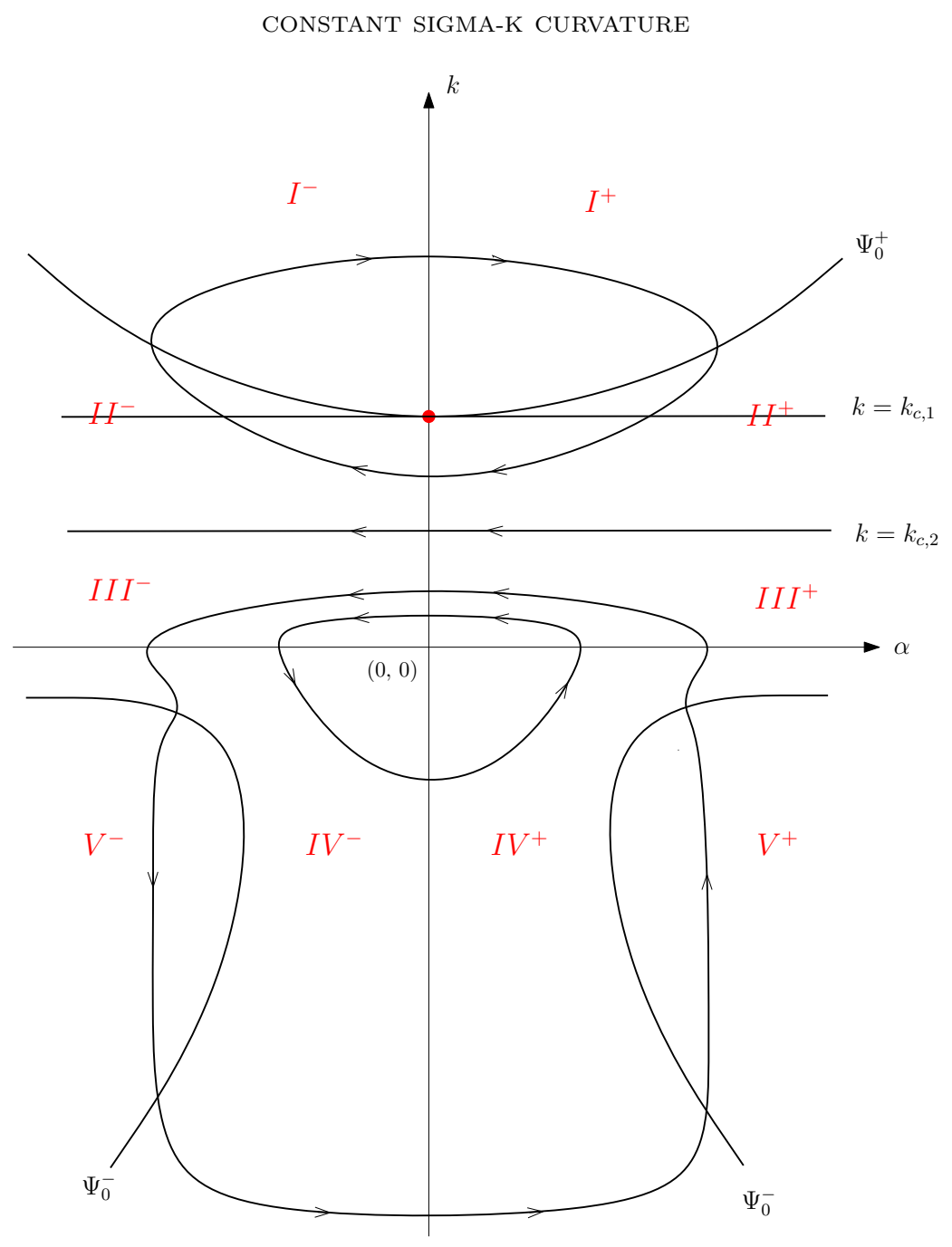

Figure 3.1: $c>0$, odd $i \geq 3$

Observe that the "discriminant"

$$
4\left(\frac{2 n-1}{i}\right)(2 n+3 i-1)>\left(\frac{-4 n+i^{2}-3 i+2}{i}\right)^{2}
$$

holds for $1 \leq i \leq 2 n-1, n \geq 2$ by elementary algebra. From (3.8) we then have the following estimate:

$$
\begin{aligned}
& \frac{\Pi}{(2 k-l)^{2} \alpha^{3}} \\
\geq & \frac{C_{1}\left(k^{2} \alpha^{2}+\alpha^{4}\right)}{(2 k-l)^{2} \alpha^{3}}=\frac{C_{1}\left(k^{2}+\alpha^{2}\right)}{(2 k-l)^{2} \alpha} \\
\geq & C_{2} \frac{k \alpha}{k^{2} \alpha}=C_{2} \frac{1}{k}
\end{aligned}
$$


for $k \geq k_{0}>k_{c, 2}$ and $\alpha>0$. Here we have used

$$
l-2 k=\frac{c}{\left(\begin{array}{c}
2 n-2 \\
i-1
\end{array}\right)} k^{1-i}-\frac{2 n+i-1}{i} k
$$

deduced from (3.5). The positive constants $C_{1}, C_{2}$ depend on $n, i, c$, and $k_{0}$. From (3.9) and 3.12 , we have

$$
\begin{aligned}
\frac{d \alpha}{d k}(k)-\frac{d \alpha}{d k}\left(k_{1}\right) & =\int_{k_{1}}^{k} \frac{d^{2} \alpha}{d k^{2}} d k \\
& \leq-C_{2} \int_{k_{1}}^{k} \frac{1}{k} d k \\
& =-C_{2} \log \frac{k}{k_{1}}
\end{aligned}
$$

for $k>k_{1} \geq k_{0}\left(>k_{c, 2}>0\right)$. The solution $(\alpha(s), k(s))$ to $(3.2)$ with $(\alpha(0), k(0))$ $=p_{0} \in I I^{+}$must hit $\Psi_{0}^{+} \cap\{\alpha>0\}$ in finite negative time. Otherwise as $s \rightarrow-\infty$, $(\alpha(s), k(s))$ stays in $I I^{+}$while $k$ goes to $+\infty$ or some finite $k_{2}$. In the first case, we let $k \rightarrow+\infty$ in 3.14 to obtain

$$
0-\frac{d \alpha}{d k}\left(k_{1}\right) \leq \lim _{k \rightarrow \infty} \inf \frac{d \alpha}{d k}(k)-\frac{d \alpha}{d k}\left(k_{1}\right) \leq-\infty,
$$

a contradiction. Here we have used the fact that $\frac{d \alpha}{d k}>0$ in $I I^{+}$. In the second case, we have $\lim _{k \rightarrow k_{2}-} \frac{d \alpha}{d k}=+\infty$ while, from 3.14 , there holds

$$
+\infty=\lim _{k \rightarrow k_{2}-} \frac{d \alpha}{d k}(k)-\frac{d \alpha}{d k}\left(k_{1}\right) \leq-C_{2} \log \frac{k_{2}}{k_{1}}
$$

a contradiction. On the other hand, it is not possible that as $s \rightarrow+\infty$, the solution $(\alpha(s), k(s))$ stays in Region $I I_{1}$. The reason is that $\lim _{k \rightarrow k_{c, 1}}+\frac{d \alpha}{d k}(k)=+\infty$ in this case while, from (3.14) (taking $k_{1}$ such that $k_{c, 1}>k_{1}>k_{c, 2}$ ), there holds

$$
+\infty=\lim _{k \rightarrow k_{c, 1}+} \frac{d \alpha}{d k}(k)-\frac{d \alpha}{d k}\left(k_{1}\right) \leq-C_{2} \log \frac{k_{c, 1}}{k_{1}}
$$

a contradiction. Observe that both $\alpha^{\prime}(s)$ and $k^{\prime}(s)$ are negative for $(\alpha(s), k(s))$ $\in I I^{+}$. The solution $(\alpha(s), k(s))$ must hit line segment $\{0\} \times\left(k_{c, 2}, k_{c, 1}\right)$ in finite positive time since it cannot hit $L_{c, 2}^{+}$(which is a solution orbit) by the uniqueness of solutions to an ODE.

Case 3. Let us look at Region $I I I^{+}$, the region surrounded by $L_{c, 2}^{+}$, line segment $\{0\} \times\left[0, k_{c, 2}\right]$, and positive $\alpha$-axis. Observe that in $I I I^{+}$, the main term (see (3.8)) $\Pi>0$ since $c>0, k>0$. Also $\alpha>0$ in $I I I^{+}$, and hence we have

$$
\frac{d^{2} \alpha}{d k^{2}}=-\frac{\Pi}{(2 k-l)^{2} \alpha^{3}}<0 .
$$

It follows that the graph $\alpha=\alpha(k)$ is convex upward, so any solution $(\alpha(s), k(s))$ to 3.2 in $I I I^{+}$must hit line segment $\{0\} \times\left[0, k_{c, 2}\right]$ in finite positive time while approaching to $\left(\alpha_{0}, 0\right)$ as $s \rightarrow-s_{1}$ for (finite) $s_{1}>0$. The reason is that it cannot hit $L_{c, 2}^{+}$by the uniqueness of solutions to an ODE since $L_{c, 2}^{+}$is a solution orbit. On 
the other hand, we have

$$
\begin{aligned}
\frac{d k}{d s}= & (l-2 k)(s) \alpha(s) \\
= & {\left[\frac{c}{\left(\begin{array}{c}
2 n-2 \\
i-1
\end{array}\right)} k^{1-i}(s)-\frac{2 n+i-1}{i} k(s)\right] \alpha(s) } \\
& \quad \text { approximates } \frac{c}{\left(\begin{array}{c}
2 n-2 \\
i-1
\end{array}\right)} k^{1-i} \alpha_{0}
\end{aligned}
$$

for $(\alpha, k)$ near $\left(\alpha_{0}, 0\right)$ and $i \geq 2$. It follows that $k^{i-1} d k$ approximates $\tilde{c} \alpha_{0} d s$ with $\tilde{c} \alpha_{0}>0$, and hence after integration, we learn that $k(s)$ goes to zero only when $s$ goes to a finite number $-s_{1}$. By (3.15) we get

$$
\lim _{s \rightarrow-s_{1}} \frac{d k}{d s}=+\infty \text {. }
$$

Next, we will discuss regions $I V^{+}$and $V^{+}$for odd $i \geq 3$ (see Figure 3.1). For even $i \geq 2$, we are done since the solution to $(3.2)$ is also symmetric with respect to the $\alpha$-axis (see Figure 3.2).

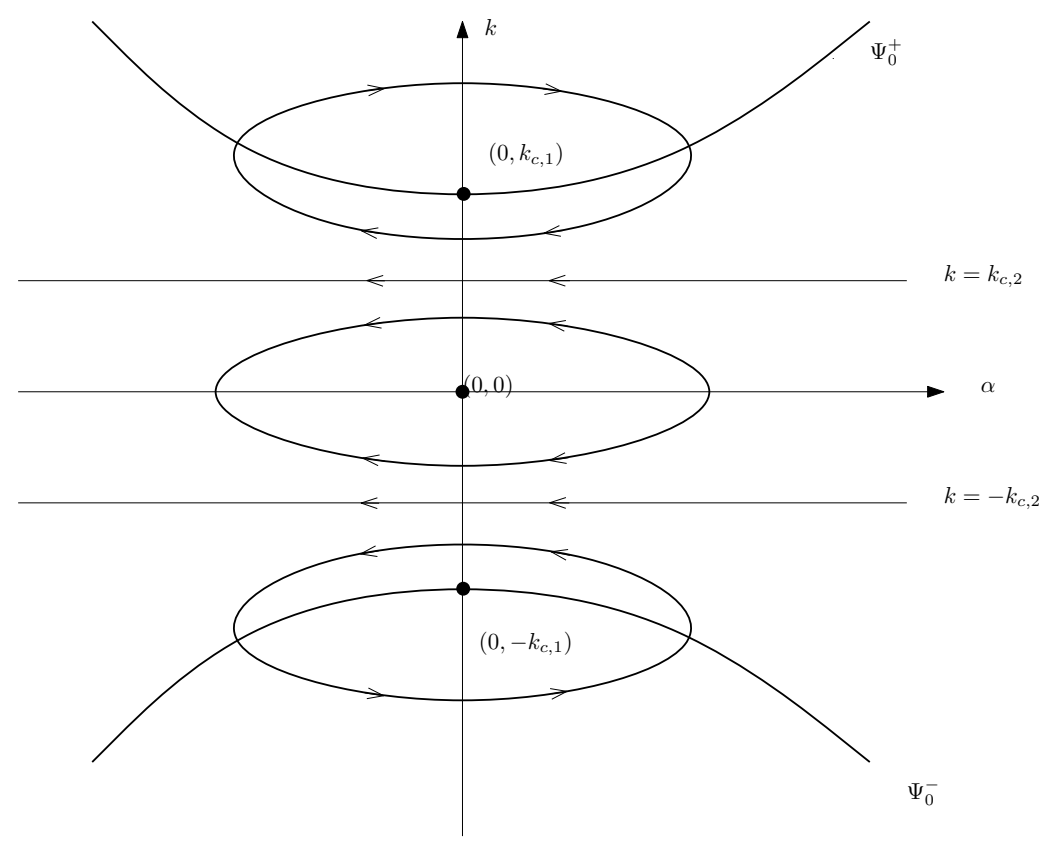

Figure 3.2: $c>0$, even $i \geq 2$

Case 4. Look at the solutions to 3.2 in region $V^{+}$: surrounded by $\Psi_{0}^{-} \cap$ $\{\alpha>0\}$, in which $\alpha^{\prime}<0$ (see Figure 3.1). We estimate the main term $\Pi$ (see (3.8)): there is a large negative $k_{0}$ such that for $k \leq k_{0}<0$ there holds

$$
\Pi \geq C_{1}\left(k^{2} \alpha^{2}+\alpha^{4}\right)
$$


where the positive constant $C_{1}$ depends on $c, k_{0}, n$, and $i$. From (3.9), 3.16), and 3.13, we then have

$$
\begin{aligned}
\frac{d^{2} \alpha}{d k^{2}} & =-\frac{\Pi}{(2 k-l)^{2} \alpha^{3}} \\
& \leq-\frac{C_{1}\left(k^{2} \alpha^{2}+\alpha^{4}\right)}{(2 k-l)^{2} \alpha^{3}} \\
& \leq-C_{1} \frac{k^{2}+\alpha^{2}}{(2 k-l)^{2} \alpha} \\
& \leq-C_{2} \frac{|k| \alpha}{k^{2} \alpha}=-C_{2} \frac{1}{|k|}
\end{aligned}
$$

for $k \leq k_{0}<0$ (may need to take larger $k_{0}$ ). Here positive constants $C_{1}, C_{2}$ depend only on $c, k_{0}, n$, and $i$. Integrating (3.17) gives

$$
\frac{d \alpha}{d k}\left(k_{0}\right)-\frac{d \alpha}{d k}(k) \leq C_{2} \log \left|\frac{k_{0}}{k}\right| .
$$

We claim the solution $(\alpha(s), k(s))$ to 3.2 with $(\alpha(0), k(0))=p_{0} \in V^{+}$with $k(0)$ $\leq k_{0}$ must hit the curve $\Psi_{0}^{-} \cap\{\alpha>0\}$ in finite negative time. Otherwise we have

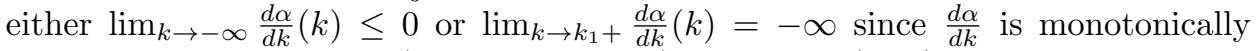
decreasing as $k$ decreases (as $s$ goes to $-\infty$ ). But from (3.18) we get

$$
\frac{d \alpha}{d k}\left(k_{0}\right) \leq \frac{d \alpha}{d k}\left(k_{0}\right)-\lim _{k \rightarrow-\infty} \frac{d \alpha}{d k}(k) \leq C_{2}(-\infty)
$$

Or

$$
-\infty=\lim _{k \rightarrow k_{1}+} \frac{d \alpha}{d k}(k)-\frac{d \alpha}{d k}\left(k_{0}\right) \leq C_{2} \log \left|\frac{k_{0}}{k_{1}}\right| .
$$

Both (3.19) and (3.20) give contradiction. Next suppose $k(0)>k_{0}$. If $k\left(s_{1}\right) \leq k_{0}$ for $s_{1}<0$, we run the above argument again to show the solution $(\alpha(s), k(s))$ must hit the curve $\Psi_{0}^{-} \cap\{\alpha>0\}$ in finite negative time. In case $k(s)>k_{0}$ for all $s<0$, we must have

$$
\lim _{k \rightarrow k_{2}+} \frac{d \alpha}{d k}(k)=-\infty \text { and } \lim _{k \rightarrow k_{2}+} \alpha(k)=+\infty
$$

for some $k_{2} \geq k_{0}$. There exists a large $\alpha_{0}$ such that for $\alpha \geq \alpha_{0}, k$ close to $k_{2}$, we have the following estimate:

$$
\begin{aligned}
\frac{d^{2} \alpha}{d k^{2}} & =-\frac{\Pi}{(2 k-l)^{2} \alpha^{3}} \\
& \leq-\frac{k^{-i} \frac{c}{\left(\begin{array}{c}
2 n-2 \\
i-1
\end{array}\right)}\left[k^{2} \alpha^{2}\left(\frac{i^{2}+2}{i}\right)+\alpha^{4}(i-1)\right]}{(2 k-l)^{2} \alpha^{3}} \\
& \leq C_{3} \alpha
\end{aligned}
$$

for some poitive constant $C_{3}$ depending on $k_{2}, \alpha_{0}, c, n, i$. Note that $k^{-i}<0$ since $k<0$ and $i$ is odd. For the first inequality in (3.22), we have dropped positive terms in $\Pi$. Multiplying 3.22 by $2 \frac{d \alpha}{d k}$ and integrating from $k$ to $k_{3}, k_{3}>k>k_{2}$, we obtain

$$
\left(\frac{d \alpha}{d k}\right)^{2}\left(k_{3}\right)-\left(\frac{d \alpha}{d k}\right)^{2}(k) \geq C_{3}\left(\alpha^{2}\left(k_{1}\right)-\alpha^{2}(k)\right) .
$$


Hence

$$
\left(\frac{d \alpha}{d k}\right)^{2}(k) \leq C_{3} \alpha^{2}(k)+C_{4}
$$

where $C_{4}=\left(\frac{d \alpha}{d k}\right)^{2}\left(k_{3}\right)-C_{3} \alpha^{2}\left(k_{1}\right)$. If $C_{4} \leq 0$, we have $\left(\frac{d \alpha}{d k}\right)^{2}(k) \leq C_{3} \alpha^{2}(k)$ from (3.23), and hence

$$
\begin{aligned}
\log \frac{\alpha(k)}{\alpha\left(k_{3}\right)} & =-\int_{k}^{k_{3}} \frac{d \alpha}{\alpha} \\
& =\int_{k}^{k_{3}} \frac{|d \alpha|}{\alpha} \leq \int_{k}^{k_{3}} \sqrt{C_{3}}|d k| \\
& =\sqrt{C_{3}}\left(k_{3}-k\right) \rightarrow \sqrt{C_{3}}\left(k_{3}-k_{2}\right)
\end{aligned}
$$

as $k \rightarrow k_{2}$. On the other hand, $\log \frac{\alpha(k)}{\alpha\left(k_{3}\right)}$ on the left hand side of 3.24 tends to $+\infty$ as $k \rightarrow k_{2}$ by (3.21). We have reached $+\infty \leq$ a finite number, a contradiction. In case $C_{4}>0$, we can absorb it in $\alpha^{2}(k)$ term for $k$ large so that $\left(\frac{d \alpha}{d k}\right)^{2}(k) \leq C_{5} \alpha^{2}(k)$ for $C_{5}>C_{3}$. A similar reasoning as above reaches a contradiction again. We have shown that in any case the solution $(\alpha(s), k(s))$ must hit the curve $\Psi_{0}^{-} \cap\{\alpha>0\}$ in finite negative time.

On the other hand, we claim $(\alpha(s), k(s))$ must hit the curve $\Psi_{0}^{-} \cap\{\alpha>0\}$ also in finite positive time. Observe that as $s$ goes to $+\infty, \alpha(s)$ decreases while $k(s)$ increases. So $(\alpha(s), k(s))$ will hit $\Psi_{0}^{-} \cap\{\alpha>0\}$ in finite positive time for topological reason.

Case 5. Let us look at solutions $\gamma(s):=(\alpha(s), k(s))$ to (3.2), which lie in Region $I V^{+}$: the region surrounded by positive $\alpha$-axis, negative $k$-axis, and the curve $\Psi_{0}^{-}$ $\cap\{\alpha>0\}$ (see Figure 3.1). Recall that $k_{0}$ is a large negative number in Case 4, such that estimate (3.17) holds for $k \leq k_{0}$. If $k\left(s_{1}\right) \leq k_{0}$ for some $s_{1}$, then $\gamma(s)$ must hit the negative $k$-axis in finite negative time since $\gamma(s)$ viewed as a graph $\alpha$ $=\alpha(k)$ is convex upward by (3.17). In the other direction, $\gamma(s)$ must hit $k=k_{0}$ line (then enters $I V^{+} \cap\left\{k>k_{0}\right\}$ ) or the curve $\Psi_{0}^{-} \cap\{\alpha>0\}$ (then enters region $\left.V^{+}\right)$in finite positive time.

In case $k\left(s_{2}\right)>k_{0}$ for some $s_{2}, \gamma(s)$ will backward hit negative $k$-axis or $k=k_{0}$ line or curve $\Psi_{0}^{-} \cap\{\alpha>0\}$ in finite negative time by topological reason (noting that $\alpha^{\prime}(s)>0$ and $k^{\prime}(s)>0$ for $\left.\gamma(s) \in I V^{+}\right)$. In the other direction, $\gamma(s)$ will hit $\Psi_{0}^{-} \cap\{\alpha>0\}$ (then enters region $V^{+}$) in finite positive time or hit positive $\alpha$-axis in infinite positive time (the same reason as shown in the discussion of Case 3 ). If both cases do not occur, then $\gamma(s)$ goes to the infinity between positive $\alpha$-axis and curve $\Psi_{0}^{-} \cap\{\alpha>0\}$. We claim this cannot happen. Observe that in $I V^{+}$, we have

$$
\begin{aligned}
\alpha^{2} & \leq k(k-l) \\
& \leq-C_{6} k^{2-i}
\end{aligned}
$$

for $k$ near 0 (noting that $i$ is odd, $i \geq 3$, and $k$ is negative). As $\alpha \rightarrow+\infty, k$ must go to 0 by the first inequality of 3.25 . Besides, the situation also forces

$$
\lim _{k \rightarrow 0^{-}} \frac{d \alpha}{d k}=+\infty
$$


On the other hand, we estimate $\Pi \geq C_{7} \alpha^{4} k^{-i}$ and hence

$$
\begin{aligned}
\frac{d^{2} \alpha}{d k^{2}} & =-\frac{\Pi}{(2 k-l)^{2} \alpha^{3}} \\
& \leq-C_{7} \frac{\alpha^{4} k^{-i}}{(2 k-l)^{2} \alpha^{3}} \\
& \leq-C_{8} \frac{\alpha k^{-i}}{\left(k^{1-i}\right)^{2}}=-C_{8} \frac{\alpha}{k^{2-i}} \\
& \leq-C_{8} \frac{\alpha^{2}}{k^{2-i}} \leq C_{9}
\end{aligned}
$$

by 3.25 for $\alpha$ large $(k$ near 0$)$. Integrating 3.27 gives

$$
\frac{d \alpha}{d k}(k)-\frac{d \alpha}{d k}(\hat{k}) \leq C_{9}(k-\hat{k})
$$

for fixed $\hat{k}$, close to 0 and $\hat{k}<k<0$. Letting $k \rightarrow 0^{-}$, we get

$$
+\infty \leq C_{9}(-\hat{k})
$$

by 3.26 , a contradiction.

The discussion for the part of $\alpha<0$ is similar since the solution $\gamma(s):=$ $(\alpha(s), k(s))$ to 3.2 is symmetric with respect to the $k$-axis, i.e., $(\alpha, k) \in \gamma$ implies $(-\alpha, k) \in \gamma$.

For convexity of solutions to $\sqrt{3.2}$ we notice the following result (see Figure 3.1 and Figure 3.2).

Corollary 3.2. Suppose we are in the same situation of Theorem 3.1. For $i \geq$ 3 odd, each solution to (3.2) in the upper half plane is convex while not convex in the lower half plane. For $i \geq 2$ even, each solution to (3.2) is always convex.

We now turn to the case $\sigma_{i, n}=c=0$.

Theorem 3.3. Assume $\sigma_{i, n}=c=0$. We are given any initial point $p_{0}=$ $\left(\alpha_{0}, k_{0}\right)$.

(a) Suppose $p_{0}=(0,0)$. Then $(\alpha(s) \equiv 0, k(s) \equiv 0)$ is the unique solution to (3.2) with $(\alpha(0), k(0))=(0,0)$. Moreover, a connected, immersed umbilic hypersurface corresponding to $\alpha \equiv 0, k \equiv 0$ is congruent with part of the hypersurface $E \times R$ where $E$ is a hyperplane of $R^{2 n}$.

(b) Suppose $p_{0}=\left(\alpha_{0}, 0\right)$ with $\alpha_{0} \neq 0$. Then we have the unique solution to (3.2) with $(\alpha(0), k(0))=\left(\alpha_{0}, 0\right)$ as follows:

$$
\alpha(s)=\frac{1}{s+\alpha_{0}^{-1}}, k(s) \equiv 0
$$

where $s \in\left(-\alpha_{0}^{-1},+\infty\right)\left(s \in\left(-\infty,-\alpha_{0}^{-1}\right)\right.$, resp. $)$ for $\alpha_{0}>0\left(\alpha_{0}<0\right.$, resp. $)$.

(c) Suppose $p_{0}=\left(\alpha_{0}, k_{0}\right)$ with $k_{0} \neq 0$. Then there is a convex curve $\gamma(s)=$ $(\alpha(s), k(s))$, solution to (3.2) with $\gamma(0)=p_{0}$ and

$$
\lim _{s \rightarrow \pm \infty} \gamma(s)=(0,0)
$$


Moreover, $\gamma$ is symmetric with respect to the $k$-axis, i.e., $(\alpha, k) \in \gamma$ implies $(-\alpha, k)$ $\in \gamma$.

Proof. (of Theorem 3.3) From 1.2, 1.3), we conclude that at each point, either $k=0$ or

$$
l=-\frac{2 n-i-1}{i} k .
$$

It is straightforward to see that the only stationary point for the ODE system 3.2 is $(\alpha, k)=(0,0)$. A connected, immersed umbilic hypersurface corresponding to $\alpha$ $\equiv 0, k \equiv 0$ is congruent with part of the hypersurface $E \times R$ where $E$ is a hyperplane of $R^{2 n}$ by Theorem 1.5 in $[6]$.

For $\alpha(0)=\alpha_{0} \neq 0, k(0)=0$, we have a (unique) solution to 3.2 as follows: $k(s) \equiv 0$,

$$
\begin{aligned}
\alpha^{\prime}(s) & =-\alpha^{2}(s), \text { and hence } \\
\alpha(s) & =\frac{1}{s+\alpha_{0}^{-1}} .
\end{aligned}
$$

The reason is as follows. Suppose $k(s) \neq 0$ for $\hat{s}<s<\hat{s}+\varepsilon$ and $k(s)=0$ for 0 $\leq s \leq \hat{s}$. Then (3.28) holds for $\hat{s}<s<\hat{s}+\varepsilon$. It follows by continuity that

$$
\begin{aligned}
l(\hat{s}) & =\lim _{s \rightarrow \hat{s}+} l(s) \\
& =\lim _{s \rightarrow \hat{s}+}\left(-\frac{2 n-i-1}{i} k(s)\right) \\
& =-\frac{2 n-i-1}{i} k(\hat{s})=0 .
\end{aligned}
$$

Now equation (3.2) with $l$ given by $(3.28)$ holds for $\hat{s} \leq s<\hat{s}+\varepsilon$. By the uniqueness of solutions to (3.2) with initial data $\alpha(0)=\alpha_{0} \neq 0, k(0)=0$, we get $k(s) \equiv 0$ and $\alpha$ is given in (3.29). We have reached a contradiction. Similarly we can argue for negative $\hat{s}$. If $\alpha_{0}>0$, we have $\alpha(s) \rightarrow 0^{+}$as $s \rightarrow+\infty$ while $\alpha(s) \rightarrow+\infty$ as $s$ $\rightarrow\left(-\alpha_{0}^{-1}\right)^{+}$. On the other hand, if $\alpha_{0}<0$, we have $\alpha(s) \rightarrow-\infty$ as $s \rightarrow\left(-\alpha_{0}^{-1}\right)^{-}$ while $\alpha(s) \rightarrow 0^{-}$as $s \rightarrow-\infty$. We have proved (a) and (b).

For (c), the solution $\gamma(s)=(\alpha(s), k(s))$ to (3.2) with $\gamma(0)=p_{0}$ has nonvanishing $k(s)$ for all $s$ by the uniqueness of solution to an ODE system. By (3.28) we can write $(3.2)$ in the following form:

$$
\begin{aligned}
k^{\prime}(s) & =-\frac{2 n+i-1}{i} k \alpha, \\
\alpha^{\prime}(s) & =\frac{2 n-1}{i} k^{2}-\alpha^{2} .
\end{aligned}
$$

From 3.8$)$ and 3.30$)$ we obtain

$$
\begin{aligned}
\Pi= & k^{2} \alpha^{2}\left(\frac{2 n-1}{i^{2}}\right)(2 n+3 i-1) \\
& +\alpha^{4}\left(\frac{2 n-i-1}{i}\right)+\left(\frac{2 n-1}{i} k^{2}-\alpha^{2}\right)^{2}
\end{aligned}
$$


since $\tilde{\sigma}_{i, n}=\frac{\sigma_{i, n}}{\left(\begin{array}{c}2 n-2 \\ i-1\end{array}\right)}=0$. From 3.9 and 3.28 we have

$$
\begin{aligned}
\frac{d^{2} \alpha}{d k^{2}} & =\frac{d}{d k}\left(\frac{\alpha^{\prime}}{k^{\prime}}\right) \\
& =-\frac{\Pi}{\left(\frac{2 n+i-1}{i} k\right)^{2} \alpha^{3}} .
\end{aligned}
$$

Observe that $\Pi>0$ by 3.31 , and hence $\frac{d^{2} \alpha}{d k^{2}}<0$ by 3.32 for $\alpha>0$. So $\gamma$, viewed as a graph $\alpha=\alpha(k)$, is convex (concave, resp.) in the $\alpha>0$ ( $\alpha<0$, resp.) half plane. Therefore $\gamma$ must hit the $k$-axis. On the other hand, $\gamma$ being symmetric with respect to the $k$-axis implies that either $\gamma$ is a periodic orbit in the $k>0(k<0$, resp.) plane or $\lim _{s \rightarrow \pm \infty} \gamma(s)=(0,0)$. In the former situation, it ends up to have a stationary point at the positive (negative, resp.) $k$-axis by a topological argument. This is impossible since $(0,0)$ is the only stationary point.

See Figure 3.3 for the $(\alpha, k)$ diagram in the case of $c=0$. We can also sketch the $(\alpha, k)$ diagram in the case of $c<0$. See the discussion in the end of Section 4 .

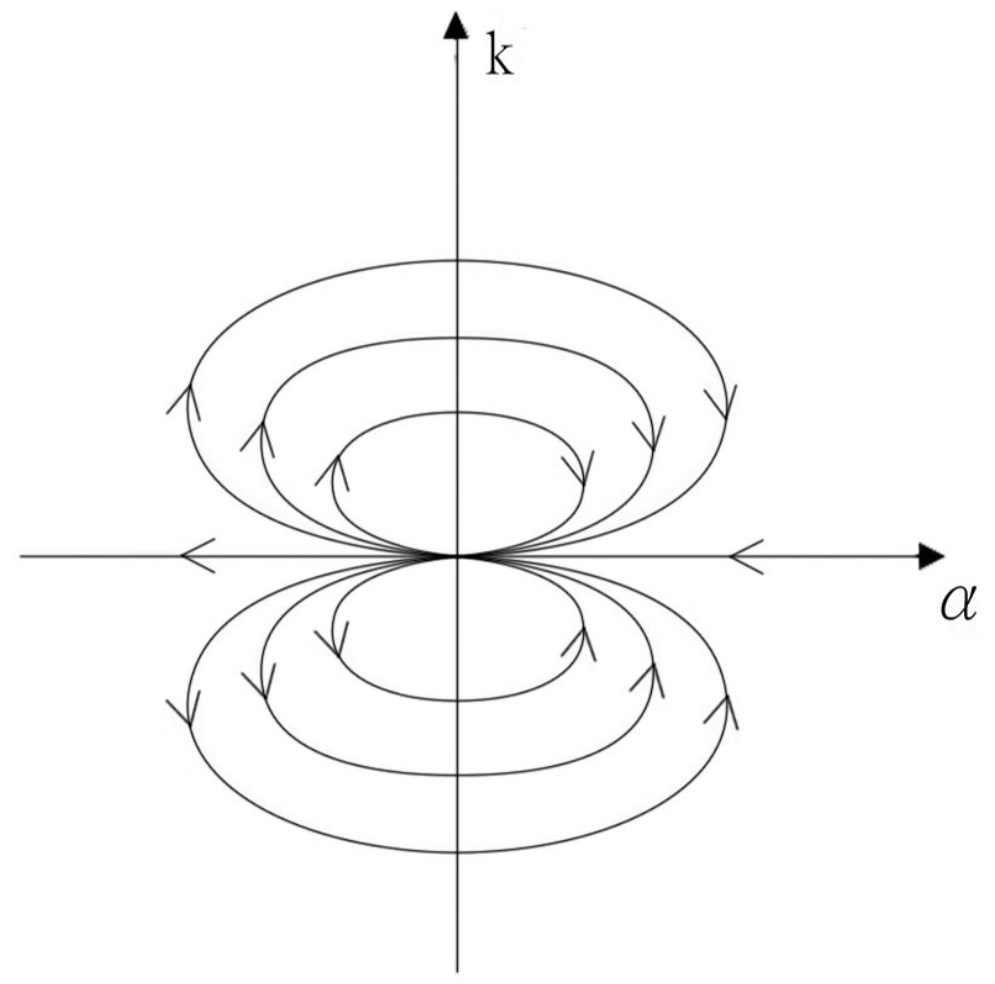

Figure 3.3: $c=0, i$ odd or even 


\section{Proof of Theorem A and Theorem C}

The proof is in the same spirit of proving Lemma B and Theorem 1.3 (or Theorem 1.4) in [6]. For completeness we will reproduce a similar reasoning here.

Proof. (of Theorem A) For $K \subset \alpha k$-plane, we define the subset $\Sigma(K) \subset \Sigma \backslash S_{\Sigma}$ (where $S_{\Sigma}$ denotes the set of singular points in $\Sigma$; see Section 1 for the definition of a singular point) by

$$
\Sigma(K):=\left\{p \in \Sigma \backslash S_{\Sigma}:(\alpha(p), k(p)) \in K\right\} .
$$

By Proposition 4.2 in [6], we learn that $k$ and $\alpha$ are constant on each leaf of the $(2 n-1)$-dimensional foliation $\mathfrak{F}$ described in Proposition 4.3 in 6]. On the other hand, $e_{n}$ is transversal to the leaves by Proposition 4.3 in [6], hence $\Sigma(K)$ is open for $K=\{(0,0)\}$ or $\left\{\left(0, k_{c, 1}\right)\right\}$ for $i$ odd $\left(\{(0,0)\}\right.$ or $\left\{\left(0, \pm k_{c, 1}\right)\right\}$, for $i$ even, resp.) or a periodic orbit or an arc-type solution to 3.2 in the upper half or lower half $\alpha k$-plane or the $k=k_{c, 2}$ line for $i$ odd ( $k= \pm k_{c, 2}$ lines for $i$ even, resp.) by Theorem 3.1. It is also clear that $\Sigma(K)$ is a closed set for such a $K$.

Note that $S_{\Sigma}$ consists of discrete (isolated singular) points by Proposition 4.1 in [6]. So $\Sigma \backslash S_{\Sigma}$ is connected and identified with $\Sigma(K)$ if $\Sigma(K) \neq \emptyset$ since $\Sigma(K)$ is open and closed.

We claim the existence of singular points. If not, $e_{n}$ is a nonvanishing global vector field. Hence the Euler number of $\Sigma$ is zero, a contradiction to the assumption. So $\Sigma$ contains at least a singular point.

Observe that $\alpha \rightarrow \pm \infty$ as regular points $p_{j}$ tend to a singular point. For $K$ being the above-mentioned sets except the $k=k_{c, 2}$ line for $i$ odd $\left(k= \pm k_{c, 2}\right.$ lines for $i$ even, resp.), $\alpha$ is bounded. Therefore the only choice of $K$ for $\Sigma(K)=\Sigma \backslash S_{\Sigma}$ is $K=$ the $k=k_{c, 2}$ line for $i$ odd ( $k= \pm k_{c, 2}$ lines for $i$ even, resp.) (if $\Sigma$ has a singular point). But on such a $K$, we have $l=2 k$. By Theorem 1.3 in [6], $\Sigma$ is congruent with a Pansu sphere $S_{\lambda}$ with $\lambda=k$.

We observe that if the horizontal normal $e_{2 n}$ changes sign, then $\sigma_{i, n}$ also changes sign for $i$ odd, but does not change sign for $i$ even.

Lemma 4.1. Suppose $e_{2 n}$ changes sign (written as $e_{2 n} \rightarrow-e_{2 n}$ ). Then $\sigma_{i, n}$ $\rightarrow-\sigma_{i, n}$ for $i$ odd while $\sigma_{i, n} \rightarrow \sigma_{i, n}$ for $i$ even.

Proof. From $e_{2 n}=J e_{n}$ and $-\nabla_{e_{n}} e_{2 n}=l e_{n}$, we have $e_{2 n} \rightarrow-e_{2 n}$ implies $e_{n} \rightarrow-e_{n}$ and $l \rightarrow-l$. By the definition of $\alpha: \alpha e_{2 n}+T \in T \Sigma$, we learn that $\alpha \rightarrow-\alpha$ if $e_{2 n} \rightarrow-e_{2 n}$. It follows that the symmetric shape operator $\mathfrak{S}$ (see (1.1)) also changes sign. So $k \rightarrow-k$. From formulas 1.2 , 1.3 , we conclude that $\sigma_{i, n}$ changes sign for $i$ odd, but does not change sign for $i$ even.

Proof. (of Theorem C) To show (a), we take $K$ to be any "solution orbit" in the $(\alpha, k)$ diagram (see Figure 3.3). Suppose $\Sigma$ is a closed, connected, umbilic (immersed) hypersurface of $H_{n}$. Following the reasoning in the proof of Theorem A, we learn that $\Sigma \backslash S_{\Sigma}$ coincides with $\Sigma(K)$ if $\Sigma(K) \neq \emptyset$. From Theorem 3.3 (a), 
$K=\{(0,0)\}$ is impossible since $\Sigma(K)$ in this case is unbounded while $\Sigma \backslash S_{\Sigma}$ is bounded (noting that $\Sigma$ is compact). For $K$ being the $\alpha^{+}$-axis $(=\{(\alpha, 0): \alpha>$ $0\})\left(\alpha^{-}\right.$-axis, resp.), $\Sigma(K)$ is also unbounded since it contains $(2 n-1)$-dimensional spheres of radius $\left(\alpha^{2}+k^{2}\right)^{-1 / 2}=|\alpha|^{-1} \rightarrow+\infty$ by Theorem B. So the case (b) in Theorem 3.3 is excluded. For case (c) in Theorem $3.3, \Sigma(K)$ is unbounded at two ends for the same reason since $\lim _{s \rightarrow \pm \infty} \gamma(s)=(0,0)$ implies spheres of radius $\left(\alpha^{2}+k^{2}\right)^{-1 / 2} \rightarrow+\infty$. We have proved (a) of Theorem C.

To show (b), we observe that $\sigma_{i, n}$ changes sign for $i$ odd if we change the sign of $e_{2 n}$ while $l$ and $k$ change sign, and $\alpha$ does not change. Moreover, when $i$ is odd, $(\alpha(s), k(s))$ is a solution to 3.2 for $\sigma_{i, n}=c<0$ if and only if $(\alpha(s),-k(s))$ is a solution to 3.2 for $\sigma_{i, n}=c>0$. So a similar reasoning as in the proof of Theorem A shows that only $K=\left\{k=-k_{-c, 1}\right\}$ is a possible "solution orbit" and $\Sigma$ must be a Pansu sphere up to a Heisenberg translation.

For $\sigma_{i, n}=c<0$ and $i$ even, we discuss and sketch the $(\alpha, k)$-diagram as follows. First observe that when $c<0, k$ is never zero for $i \geq 2$. From 1.2 we get

$$
l=\frac{c}{\left(\begin{array}{c}
2 n-2 \\
i-1
\end{array}\right)} k^{1-i}-\frac{2 n-i-1}{i} k
$$

for $2 \leq i \leq 2 n-1$. By (4.1) we compute

$$
\begin{aligned}
l-2 k & =k\left[\frac{c}{\left(\begin{array}{c}
2 n-2 \\
i-1
\end{array}\right)} k^{-i}-\frac{2 n-i-1}{i}-2\right], \\
k(k-l) & =k^{2}\left[1-\frac{c}{\left(\begin{array}{c}
2 n-2 \\
i-1
\end{array}\right)} k^{-i}+\frac{2 n-i-1}{i}\right] .
\end{aligned}
$$

Since $c<0$ and (hence) $k \neq 0$, we have $k(k-l)>0$ by the second equality of (4.2). It follows that there are no stationary points (where $k^{\prime}=0, \alpha^{\prime}=0$ ) for the ODE system 3.2 in this situation ( $c<0$ and $i$ even). Recall that $\Psi_{0}^{ \pm}$in $(\alpha, k)$-plane is defined by $k^{2}-\alpha^{2}-k l=0$ (where $\alpha^{\prime}=0$ ), in $k \gtrless 0$ regions, resp.. For the case $i$ $=2, \Psi_{0}^{ \pm}$is described by the following hyperbolic curve:

$$
\left(1+\frac{2 n-i-1}{i}\right) k^{2}-\alpha^{2}=\frac{c}{\left(\begin{array}{c}
2 n-2 \\
i-1
\end{array}\right)} .
$$

On the other hand, for $i \geq 4, \Psi_{0}^{ \pm}$is a curve of different type. We have sketched the solution diagram in Figure 4.1 and Figure 4.2 for these two cases.

We say a hypersurface $\Sigma$ can be completed to a closed hypersurface $\bar{\Sigma}$ if $\bar{\Sigma}$ contains $\Sigma$ and $\bar{\Sigma}$ is closed, i.e., compact with no boundary.

Proposition 4.2. Given a solution curve $\gamma$ in Figure 4.2: $c<0$, even $i \geq 4$ (note that $k \equiv 0$ ( $\alpha$-axis) is not a solution curve), suppose there corresponds an umbilic hypersurface $\Sigma$ with its $(\alpha(s), k(s))$ curve being $\gamma$. Then $\Sigma$ can be completed to a closed, rotationally invariant hypersurface $\bar{\Sigma}$ up to a Heisenberg translation and $\bar{\Sigma}$ is $C^{2}$ smooth at its two singular points. 


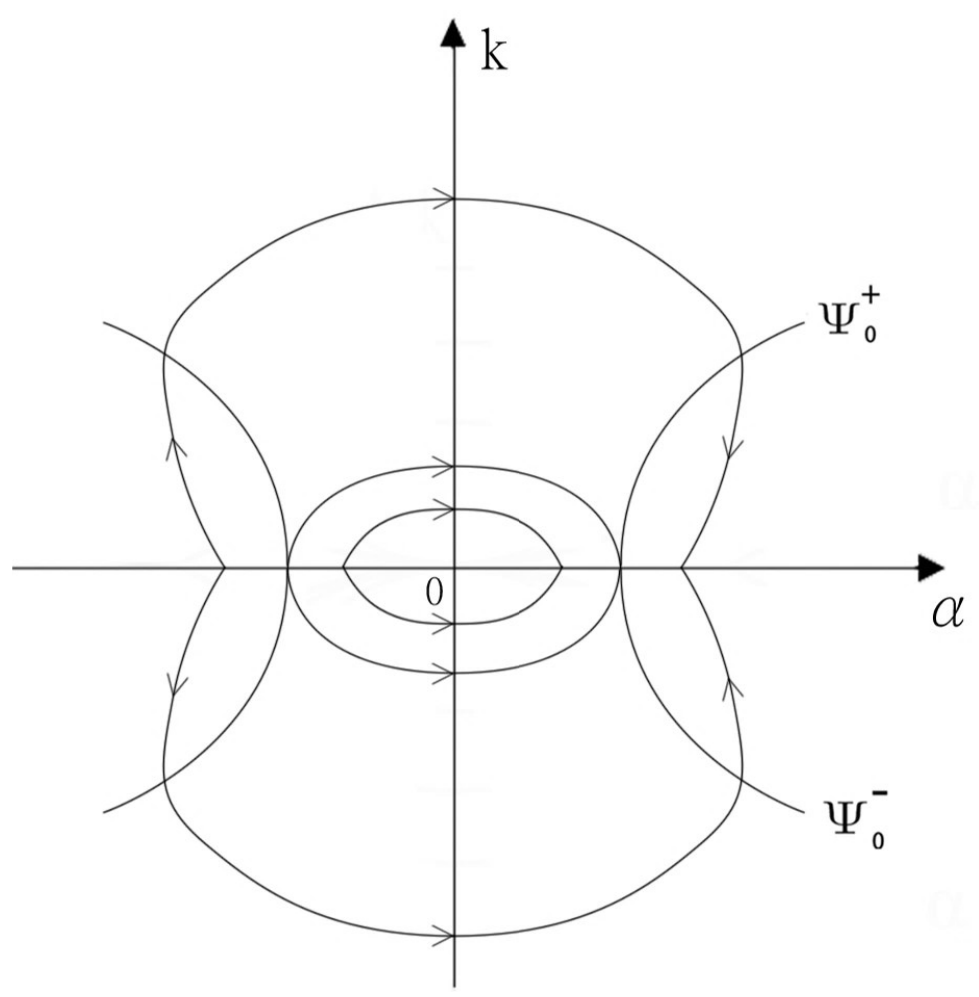

Figure 4.1: $c<0, i=2$

Proof. By Theorem B (c), $\Sigma$ is congruent with part of a rotationally invariant hypersurface $\Sigma^{\prime}$ and the radius $r=\frac{1}{\sqrt{\alpha^{2}+k^{2}}}$ of leaves in the associated foliation strictly decreases to 0 as $\alpha$ goes to $\pm \infty$ (while $s \rightarrow \pm \infty$ ). Write $\Sigma^{\prime}$ as a graph $t=$ $g_{+}(r)\left(g_{-}(r)\right.$, resp. $), g_{ \pm} \in C^{\infty}$, for $r:=\left[\sum_{j=1}^{n}\left(x_{j}^{2}+y_{j}^{2}\right)\right]^{1 / 2}$ close to 0 and $\alpha$ near $+\infty$ ( $\alpha$ near $-\infty$, resp.). For $\vec{F}=-y_{j} \frac{\partial}{\partial x_{j}}+x_{j} \frac{\partial}{\partial y_{j}}$, we compute

$$
\nabla g_{ \pm}+\vec{F}=\left(g_{ \pm}^{\prime}(r) \frac{x_{j}}{r}-y_{j}\right) \frac{\partial}{\partial x_{j}}+\left(g_{ \pm}^{\prime}(r) \frac{y_{j}}{r}+x_{j}\right) \frac{\partial}{\partial y_{j}}
$$

It follows that

$$
\frac{1}{|\alpha|}=\left|\nabla g_{ \pm}+\vec{F}\right|=\sqrt{\left(g_{ \pm}^{\prime}(r)\right)^{2}+r^{2}} \rightarrow 0
$$

as $s \rightarrow \pm \infty$. So $r \rightarrow 0$ and $g_{ \pm}^{\prime}(r) \rightarrow 0$. Therefore $t$ is bounded as $r \rightarrow 0$ and $\lim _{r \rightarrow 0} g_{ \pm}(r)$ exists, denoted as $g_{ \pm}(0)$. Also $g_{ \pm}$is $C^{1}$ smooth at $r=0$ (singular points). So $\Sigma^{\prime}$ can be completed to a closed rotationally invariant hypersurface $\bar{\Sigma}$ containing two (singular) points $\left(r=0, g_{ \pm}(0)\right)$. 


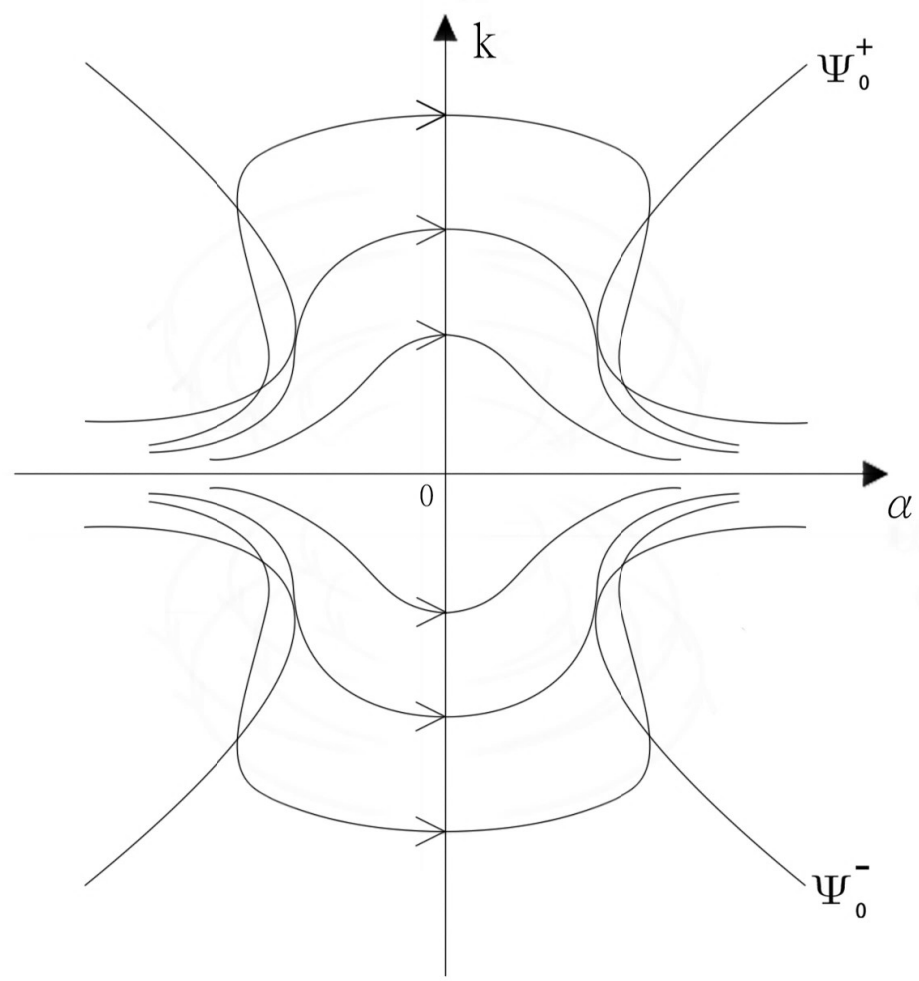

Figure 4.2: $c<0$, even $i \geq 4$

From the formula in 4.3 and $r=\frac{1}{\sqrt{\alpha^{2}+k^{2}}}$, we compute

$$
\begin{aligned}
\left(\frac{g_{ \pm}^{\prime}(r)}{r}\right)^{2}+1 & =\frac{1}{\alpha^{2} r^{2}} \\
& =\frac{\alpha^{2}+k^{2}}{\alpha^{2}} \rightarrow 1
\end{aligned}
$$

as $\alpha \rightarrow \pm \infty, k \rightarrow 0$ (while $s \rightarrow \pm \infty$ ). It follows from 4.4 that $\frac{g_{ \pm}^{\prime}(r)}{r} \rightarrow 0$ as $r \rightarrow 0$. Hence $g_{ \pm}^{\prime \prime}(0)=0$. A routine verification shows that $\Sigma$ defined by $g_{ \pm}$is $C^{2}$ smooth at two (singular) points $\left(r=0, g_{ \pm}(0)\right)$, resp..

We remark that the above hypersurface $\bar{\Sigma}$ in Proposition 4.2 is not a Pansu sphere on which $k$ is a constant.

For the case $c<0$ and odd $i \geq 3$, the $(\alpha, k)$ diagram is the reflection of Figure 3.1 with respect to the $\alpha$-axis by Lemma 4.1 (see Figure 4.3). 


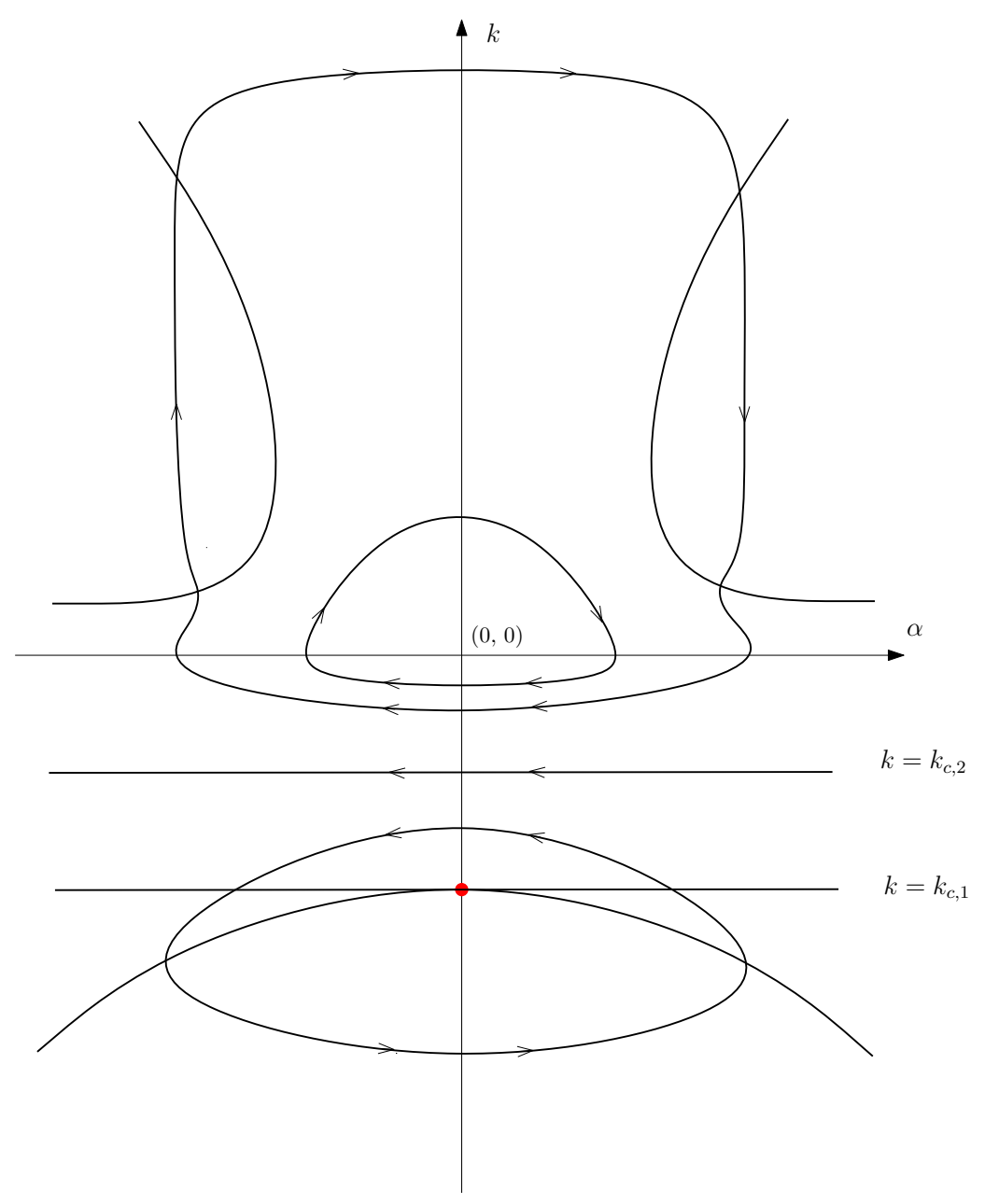

Figure 4.3: $c<0$, odd $i \geq 3$

5. Appendix A: Closed form of solutions to 3.2

Write $(3.2)$ in terms of differential form as follows:

$$
\left(k^{2}-k l-\alpha^{2}\right) d k+(2 k-l) \alpha d \alpha=0
$$

where we recall (see (3.3)) that

$$
l=\frac{c}{\left(\begin{array}{c}
2 n-2 \\
i-1
\end{array}\right) k^{i-1}}-\frac{2 n-i-1}{i} k .
$$

Try to find an integrating factor $f(k)$ for 5.1. Let $\zeta:=\alpha^{2}$. That is, there holds

$$
d\left\{f(k)\left[\left(k^{2}-k l-\zeta\right) d k+(2 k-l) \frac{d \zeta}{2}\right]\right\}=0 .
$$

It follows that

$$
\frac{\partial(f(k) \zeta)}{\partial \zeta}+\frac{\partial}{\partial k}\left(f(k) \frac{2 k-l}{2}\right)=0
$$


So $f(k)$ satisfies the following ODE:

$$
2 f(k)+\frac{d}{d k}(f(k)(2 k-l))=0
$$

Let $g(k):=f(k)(2 k-l)$. We can rewrite 5.4 as

$$
\frac{d g(k)}{d k}=-\frac{2 g(k)}{2 k-l}
$$

and get the solution:

$$
g(k)=\exp \left\{-\int \frac{2}{2 k-l} d k\right\}
$$

From (5.3) and (5.4), we obtain

$$
d\left(\int f(k)\left(k^{2}-k l\right) d k+\frac{\zeta g(k)}{2}\right)=0
$$

Thus we have

$$
\int f(k)\left(k^{2}-k l\right) d k+\frac{\alpha^{2} g(k)}{2}=C_{1}
$$

for some constant $C_{1}$. We can then express $\alpha$ in terms of $k$ as follows:

$$
\alpha^{2}=\frac{2 C_{1}}{g(k)}-\frac{2}{g(k)} \int f(k)\left(k^{2}-k l\right) d k
$$

Substituting 5.2 into 5.5 gives

$$
\begin{aligned}
g(k) & =\exp \left\{\frac{-2}{i} \int \frac{d\left(k^{i}\right)}{\left.\left(\frac{2 n-i-1}{i}\right) k^{i}-\frac{c}{\left(\begin{array}{c}
2 n-2 \\
i-1
\end{array}\right)}\right\}}\right. \\
& =C_{2} \exp \left\{\frac{-2}{2 n+i-1} \log \left|k^{i}-\frac{i}{2 n+i-1} \frac{c}{\left(\begin{array}{c}
2 n-2 \\
i-1
\end{array}\right)}\right|\right.
\end{aligned}
$$

for some constant $C_{2}>0$, and hence we have

$$
g(k)=C_{2}\left|k^{i}-\frac{i}{2 n+i-1} \frac{c}{\left(\begin{array}{c}
2 n-2 \\
i-1
\end{array}\right)}\right|^{\frac{-2}{2 n+i-1}} .
$$

Next by 5.7 we compute

$$
\begin{aligned}
& \int f(k)\left(k^{2}-k l\right) d k \\
= & \int g(k) \frac{k^{2}-k l}{2 k-l} d k \\
= & C_{2} \int\left\{\left|k^{i}-\frac{i}{2 n+i-1} \frac{c}{\left(\begin{array}{c}
2 n-2 \\
i-1
\end{array}\right)}\right|^{\frac{-2}{2 n+i-1}}\left(k^{i}-\frac{i}{2 n+i-1} \frac{c}{\left(\begin{array}{c}
2 n-2 \\
i-1
\end{array}\right)}\right)^{-1}\right. \\
& \left.\frac{k}{2 n+i-1}\left[(2 n-1) k^{i}-\frac{c}{\left(\begin{array}{c}
2 n-2 \\
i-1
\end{array}\right)} i\right]\right\} d k .
\end{aligned}
$$

In view of 5.7 and $5.8,5.6$ is a closed form of solutions $(\alpha(s), k(s))$ to 3.2 . 


\section{REFERENCES}

[1] L. Ambrosio, F. Serra Cassano, and D. Vittone, Intrinsic regular hypersurfaces, J. reine angew. Math., 564 (2003) 63-83.

[2] F. Bigolin and F. Serra Cassano, Distributional solutions of Burgers' equation and intrinsic regular graphs in Heisenberg groups, J. Math. Anal. Appl. 366 (2010) 561-568.

[3] J.-M. Bony, Principe du maximum, inégalité de Harnack et unicité du problème de Cauchy pour les opérateurs elliptiques dégénérés, Annales de l'institut Fourier, tome 19 (1969) 277304.

[4] L. Capogna, D. Danielli, N. Garofalo, The geometric Sobolev embedding for vector fields and the isoperimetric inequality, Comm. Anal. Geom., 2 (1994) 203-215.

[5] J.-H. Cheng, Surfaces of prescribed p-mean curvature in the Heisenberg group, Proceedings ICCM, 2007, Hangzhou.

[6] J.-H. Cheng, H.-L. Chiu, J.-F. Hwang, and P. Yang, Umbilicity and characterization of Pansu spheres in the Heisenberg group, to appear in Crelle's journal.

[7] J.-H. Cheng and J.-F. Hwang, Properly embedded and immersed minimal surfaces in the Heisenberg group, Bull. Aus. Math. Soc., 70 (2004) 507-520.

[8] J.-H. Cheng and J.-F. Hwang, Variations of generalized area functionals and p-area minimizers of bounded variation in the Heisenberg group, Bulletin of the Institute of Mathematics, Academia Sinica, New Series, 5 (2010) 369-412.

[9] J.-H. Cheng and J.-F. Hwang, Uniqueness of generalized p-area minimizers and integrability of a horizontal normal in the Heisenberg group, Calculus of Variations and PDEns, 50 (2014) 579-597.

[10] J.-H. Cheng, J.-F. Hwang, A. Malchiodi, and P. Yang, Minimal surfaces in pseudohermitian geometry, Ann Scuola Norm. Sup. Pisa Cl. Sci. (5) Vol. IV (2005) 129-177.

[11] J.-H. Cheng, J.-F. Hwang, A. Malchiodi, and P. Yang, A Codazzi-like equation and the singular set for $C^{1}$ smooth surfaces in the Heisenberg group, Journal fur die reine und angewandte Mathematik, 671 (2012) 131-198

[12] J.-H. Cheng, J.-F. Hwang, and P. Yang, Existence and uniqueness for p-area minimizers in the Heisenberg group, Math. Annalen, 337 (2007) 253-293.

[13] J.-H. Cheng, J.-F. Hwang, and P. Yang, Regularity of $C^{1}$ smooth surfaces with prescribed p-mean curvature in the Heisenberg group, Math. Annalen, 344 (2009) 1-35.

[14] H.-L. Chiu and S.-H. Lai, The fundamental theorem for hypersurfaces in Heisenberg groups, Cal. Var. and P.D.E., 54 (2015) 1091-1118.

[15] D. Danielli, N. Garofalo, and D.-M. Nhieu, Minimal surfaces, surfaces of constant mean curvature and isoperimetry in Carnot groups, preprint, 2001.

[16] B. Franchi, R. Serapioni, and F. Serra Cassano, Rectifiability and perimeter in the Heisenberg group, Math. Ann., 321 (2001) 479-531.

[17] N. Garofalo and D.-M. Nhieu, Isoperimetric and Sobolev inequalities for CarnotCaratheodory spaces and the existence of minimal surfaces, Comm. Pure Appl. Math., 49 (1996) 1081-1144.

[18] D. Gilbarg and N. S. Trudinger, Elliptic partial differential equations of second order, 2nd ed., G.M.W. 224, Springer-Verlag, 1983.

[19] G. Leonardi and S. Masnou, On the isoperimetric problem in the Heisenberg group $H^{n}$, Ann. Mat. Pura Appl. (4), No.4, 184 (2005) 533-553.

[20] G. Leonardi and S. Rigot, Isoperimetric sets on Carnot groups, Houston J. Math., No.3, 29 (2003) 609-637.

[21] U. Massari and M. Miranda, Minimal surfaces of codimension one, North-Holland, Amsterdam-New York-Oxford, 1984.

[22] R. Monti and M. Rickly, Convex isoperimetric sets in the Heisenberg group, Ann. Sc. Norm. Super. Pisa Cl. Sci. (5), No.2, 8 (2009) 391-415.

[23] P. Pansu, Une inegalite isoperimetrique sur le groupe de Heisenberg, C. R. Acad. Sci. Paris Sér. I Math., No.2, 295 (1982) 127-130.

[24] S. D. Pauls, Minimal surfaces in the Heisenberg group, Geometric Dedicata, 104 (2004) 201231.

[25] M. Ritoré, A proof by calibration of an isoperimetric inequality in the Heisenberg group $H^{n}$, preprint. 
[26] M. Ritoré and C. Rosales, Rotationally invariant hypersurfaces with constant mean curvature in the Heisenberg group $H^{n}$, The Journal of Geometric Analysis, No.4, 16 (2006) 703-720.

[27] M. Ritoré and C. Rosales, Area-stationary surfaces in the Heisenberg group $H^{1}$, Advances in Math., 219 (2008) 633-671.

[28] F. Serra Cassano and D. Vittone, Graphs of bounded variation, existence and local boundedness of nonparametric minimal surfaces in Heisenberg groups, preprint.

Institute of Mathematics, Academia Sinica, Taipei and National Center for TheoRetical Sciences, Taiwan, R.O.C.

E-mail address: cheng@math.sinica.edu.tw

Department of Mathematics, National Central University, Chung Li, 32054, Taiwan, R.O.C.

E-mail address: hlchiu@math.ncu.edu.tw

Institute of Mathematics, Academia Sinica, Taipei, Taiwan, R.O.C.

E-mail address: majfh@math.sinica.edu.tw

Department of Mathematics, Princeton University, Princeton, NJ 08544, U.S.A.

E-mail address: yang@Math.Princeton.EDU 